Article

\title{
Evaluation of Volatile Compounds during Ageing with Oak Chips and Oak Barrel of Muscat Ottonel Wine
}

\author{
Anamaria Călugăr ${ }^{1,2}$, Teodora Emilia Coldea ${ }^{3, * \mathbb{C}}$, Carmen Rodica Pop ${ }^{4}$, Tiberia Ioana Pop ${ }^{5}$,
} Anca Cristina Babeș ${ }^{1,2}$, Claudiu Ioan Bunea ${ }^{1,2}$, Mihail Manolache ${ }^{1}$ and Emese Gal ${ }^{6, *}$

1 Faculty of Horticulture, University of Agricultural Sciences and Veterinary Medicine Cluj-Napoca, 3-5 Manastur Street, 400372 Cluj-Napoca, Romania; anamaria.calugar@usamvcluj.ro (A.C.); ancababes@usamvcluj.ro (A.C.B.); claudiu.bunea@usamvcluj.ro (C.I.B.); mihail.manolache@yahoo.com (M.M.)

2 Viticulture and Oenology Department, Advanced Horticultural Research Institute of Transylvania, Faculty of Horticulture, University of Agricultural Sciences and Veterinary Medicine Cluj-Napoca, 3-5 Manastur Street, 400372 Cluj-Napoca, Romania

3 Department of Food Engineering, Faculty of Food Science and Technology, University of Agricultural Sciences and Veterinary Medicine Cluj-Napoca, 3-5 Manastur Street, 400372 Cluj-Napoca, Romania

4 Department of Food Science, Faculty of Food Science and Technology, University of Agricultural Sciences and Veterinary Medicine Cluj-Napoca, 3-5 Manastur Street, 400372 Cluj-Napoca, Romania; carmen-rodica.pop@usamvcluj.ro

5 Department of Technical and Soil Sciences, Faculty of Agriculture, University of Agricultural Sciences and Veterinary Medicine Cluj-Napoca, 3-5 Manastur Street, 400372 Cluj-Napoca, Romania; tiberia.pop@usamvcluj.ro

6 Faculty of Chemistry and Chemical Engineering, Babeș-Bolyai University, 11 Arany Janos Street, 400028 Cluj-Napoca, Romania

* Correspondence: teodora.coldea@usamvcluj.ro (T.E.C.); emese@chem.ubbcluj.ro (E.G.); Tel.: +40-746-170337 (T.E.C.); +40-726-105405 (E.G.)

Received: 15 July 2020; Accepted: 12 August 2020; Published: 17 August 2020

\begin{abstract}
The aim of this work was to compare the variations of alcohols compounds in white wine Muscat Ottonel variety aged in the presence of untoasted oak chips, toasted oak chips and untoasted barrel, considering three ageing periods-30, 60, and 90 days. The liquid-liquid extraction and gas chromatography coupled to mass spectrometry were used to compare the concentrations of the volatile constituents of Muscat Ottonel wines. A total of 51 volatile compounds were quantified. Alcohols, terpenic and carboxylic acids decreased with ageing time, whereas esters, lactones, and phenolic compounds increased due esterification processes. The chips toast level, method, and duration of ageing, significantly influenced the content of aromatic compounds. Partial least squares regression (PLS-R) clearly discriminated the initial wine and also the wines aged with toasted and untoasted medium. The compounds (alcohols and terpenes) that impart distinctive aroma of Muscat Ottonel were enhanced by untoasted medium. Light toasted oak chips enhanced wood volatile components (acetovanillone and p-vinyl guaiacol). This study provides important scientific results on the ageing of Muscat Ottonel wines with practical economic benefits to winemakers. Alternative less expensive ageing methods and improved control on the wood components extraction process, may contribute to obtaining high-quality wines.
\end{abstract}

Keywords: Muscat Ottonel; ageing process; extraction methods; volatile compounds 


\section{Introduction}

With beer and wine ranking top consumer preferences [1], the past few years determined the wine strategy orientation, mostly on wine quality, with a focus on market expectations and its continuous diversification [2]. As red wine is becoming more popular among health-conscious consumers [3], white wine consumers are segmented, depending on the level of wine knowledge. Consumers with higher wine knowledge like and consume more specific wine assortments, proving the importance of tailoring producers marketing to specific demographic groups [4]. A recent study proved that consumer acceptance is unrelated to the wine production method. The highest consumer preference score was for wines exhibiting the most intense toasty, yeasty notes, with lower interest for the producing procedure [5].

The wood type used for wood barrels is mainly based on the producer's choice, mostly justified by economic factors. The continuous growing of wood barrels need in wine and spirit drinks industries, along with the increase of costs due to the limitation of forest wood exploitation, and handling wooden barrels [6], conducted some producers to low-cost alternatives, such as the using in cooperage of wood fragments of different wood origin. Considering the economic point of view, the two practices-wood barrels versus wooden chips using-consist of extremely different costs, especially when exclusive new barrels are used [7], reflected in the final price of the product. It is worth also mentioning the advantage of wood fragments use, considering it as a sustainable procedure, for both economic and environmental perspectives [8]. Even though many studies focused lately the quality impact of chestnut, oak, acacia, ash, cherry, or fig wooden fragments and barrels on aged wine and distilled beverages [6,9-13], the International Organization of Vine and Wine (OIV) regulates the using only oak and chestnut wooden chips for the ageing of these beverages.

Considering the volatile profile of white wines, this was intensively evaluated especially through gas chromatography-mass spectrometry (GC-MS) $[14,15]$ and gas chromatography coupled with flame ionization detector [16]. The final volatile profile of aged white wine is influenced by the barrels' wood provenience [16]. French oak barrels help a higher extraction of phenolic compounds that might negatively affect the volatile and sensory profiles of white wine. Contrarily, the same study showed a very low effect of barrel type on the concentration of esters, but wine characteristics being affected by higher alcohols and their esters.

To our best knowledge, no other study focused on the assessment of aged white wine Muscat Ottonel. Three ageing periods were considered. We choose these variations in order to find a low-cost and sustainable alternative for the ageing of white wine. Investigations in this field focused mainly on the impact of toasted barrels or toasted wooden chips on aged wines and beverages quality.

Specific floral and fresh notes of the white wine Muscat Ottonel are given by the alcohols and terpenes. When aged, those characteristic notes could be masked by the release of the compounds from wood. Generally, aromatic wines need a special ageing procedure, to avoid the roasted and bitter notes of furanic compounds from toasted wood.

The aim of this work was to compare the variations of volatile compounds in white wine Muscat Ottonel variety aged in the presence of untoasted oak chips, toasted oak chips, and untoasted barrel, considering three ageing periods.

\section{Materials and Methods}

\subsection{Winemaking Process}

Vitis vinifera L. cv. Muscat Ottonel white grapes were obtained from the Lechinta Vineyard, a Romanian winemaking region, at their optimal ripening stage ( $215 \mathrm{~g} / \mathrm{L}$ sugar, $5.21 \mathrm{~g} / \mathrm{L}$ expressed as sulphuric acid and $90 \mathrm{~g}$ weight of 100 berries), in 2017. The wines were processed at Teaca winery, following the traditional aromatic white winemaking procedure. The marc obtained after crushing the grapes was subjected to maceration-fermentation at $14{ }^{\circ} \mathrm{C}$, for a period of $8-10 \mathrm{~h}$. The marc was pressed using a pneumatic press, Vaslin-Bucher XPro 5, France, and the wine obtained was transferred 
to fermentation tanks for completing the alcoholic fermentation. The commercial active yeasts Fermactive ${ }^{\circledR}$ Primeur and Fermactive ${ }^{\circledR}$ Activateur Complex (Sodinal ${ }^{\circledR}$ Wine and Beverage Division of AVeX Group, Paris, France) were used. Wines were supplied with $15 \mathrm{mg} / \mathrm{L}$ of sulphur dioxide and divided into 3 stainless-steel tanks of $5000 \mathrm{~L}$ of the finished wine, at the end of the fermentation processes. Experimental variants consisted in ageing finished wines for 30, 60, and 90 days with two types of oak chips (untoasted and light toasted) and in untoasted barrel $(225 \mathrm{~L})$. The temperature of ageing was $12{ }^{\circ} \mathrm{C}$ in the cellar, without light. Each experimental variant was reproduced in triplicate generating in total 6 demijohns of $50 \mathrm{~L}$ ( 3 demijohns wines aged with untoasted oak chips- $4 \mathrm{~g} / \mathrm{L}$ and 3 demijohns for wines aged with light toasted oak chips $-4 \mathrm{~g} / \mathrm{L}$ ) and 3 untoasted barrels for barrel-aged wines. Both the oak chips and barrels were obtained from Quercus robur. The oak chips were provided by Arobois ${ }^{\circledR}$ (Gagnac sur Cère, France) with dimensions of $0.5 \mathrm{~cm}$ width, $1.5 \mathrm{~cm}$ length, and $0.2 \mathrm{~cm}$ thickness. The barrels were provided by Transylvania Bois ${ }^{\circledR}$ (Sighetu Marmației, Romania). After each period of ageing, wine samples were stored at $4{ }^{\circ} \mathrm{C}$ until the moment of analysis.

\subsection{Standard Chemical Analysis of Wine}

The $\mathrm{pH}$, total and volatile acidity, alcohol content (\%vol. alcohol), reducing sugar, total, and free $\mathrm{SO}_{2}$ in Muscat Ottonel white wines were conducted based on previous experiments [17]. The oenological analysis was made for initial wine and for aged wines samples (30, 60, and 90 days).

\subsection{Extraction of Volatile Compounds}

In the literature, there are several analytical techniques described for the extraction of wine flavour compounds $[18,19]$. Liquid-liquid extraction combined with ultrasound extraction is one of the most commonly used procedures. The convenience of this method is that all volatile compounds can be analysed in one extraction step. Although this is a time-consuming method, is still the reference technique for the extraction of volatile compounds from wine. The volatile compounds have a high partition coefficient to the organic phase. Different organic solvents were used in the extraction procedure (hexane/ethyl ether mixture, tert-butyl methyl ether, petrol ether, dichloromethane, etc.), from these solvents dichloromethane has been found to be more suitable for extracting volatile compounds from wine.

All samples were kept at $4{ }^{\circ} \mathrm{C}$ before the analysis. The reference standard, 2-phenyl ethanol, and 1-octanol (used as internal standard, IS) were supplied from Fluka ${ }^{\circledR}$ (Buchs, Switzerland). Dichloromethane, used for extraction of volatiles, was purchased from Merck ${ }^{\circledR}$ (Darmstadt, Germany). The standard stock solutions were prepared by dissolving $10 \mathrm{mg}$ of each reference compound in $10 \mathrm{~mL}$ of dichloromethane.

The protocol was adapted based on previous work [20]. To a volume of $50 \mathrm{~mL}$ of wine spiked with 1-octanol (614 $\mu \mathrm{g} / \mathrm{L}$ concentration) internal standard was added $10 \mathrm{~mL}$ of dichloromethane and placed in Erlenmeyer flask equipped with a ground stopper. The extraction was carried out under continuous stirring in an ice bath for $1 \mathrm{~h}$. Then the mixture was kept for $30 \mathrm{~min}$ in an ultrasonic bath at the same temperature, to avoid possible formation of an emulsion. After separation, the organic layer was dried on $\mathrm{Na}_{2} \mathrm{SO}_{4}$, evaporated under a nitrogen stream to approximately $200 \mu \mathrm{L}$ volume of the extract. From this solution, $1 \mu \mathrm{L}$ was injected into the GC-MS system. All extractions were carried out in duplicate.

\subsection{GC-MS Analysis}

Analysis of wine volatile compounds was carried out using a Shimadzu QP 2010 PLUS Mass Spectrometer coupled with Gas Chromatograph (Kyoto, Japan) equipped with a Carbowax type column from Agilent, with a dimension $30 \mathrm{~m} \times 0.32 \mathrm{~mm}$ ID and $0.50 \mu \mathrm{m}$ film thicknesses. The carrier gas was He (6.0) with a flow rate $1.7 \mathrm{~mL} / \mathrm{min}$. The working parameters were: injector temperature $220^{\circ} \mathrm{C}$, the ion source temperature $220^{\circ} \mathrm{C}$, and the interface temperature $220^{\circ} \mathrm{C}$. The column temperature program was conducted as follows: $40^{\circ} \mathrm{C}$ was the initial temperature for $5 \mathrm{~min}$, increasing at a rate of 
$4{ }^{\circ} \mathrm{C} / \mathrm{min}$ to $220^{\circ} \mathrm{C}$, and holding $220^{\circ} \mathrm{C}$ for $15 \mathrm{~min}$. The electron impact (EI) was set at $70 \mathrm{eV}$. A mass range of 35-500 $\mathrm{m} / \mathrm{z}$ was recorded at one scan per second.

A commercial solution of n-alkanes (C5-C27) in hexane was used under the same conditions using GC-MS, for calculation of the retention indices. The results from previous studies suggest that alcohols are the dominant constituents. The 1-octanol was chosen as the most suitable as internal standard, because it is similar to the expected ones, but not present in wine. In order to quantify the volatile compounds present in the wines, the relative peak area (in the total ion chromatogram) of each analyte from the wine to the internal standard was compared to those obtained for the standards, taking into account the dilution of the samples. The concentration of the volatile compounds was obtained using the calibration curve of 2-phenylethanol $(64-12,900 \mu \mathrm{g} / \mathrm{mL})$ [21]. Peak identification was carried out by analogy of mass spectra with those of the mass library (National Institute of Standards and Technology-NIST MS database) and comparing the calculated retention indices with those published in the literature.

\subsection{Statistical Analyses}

Multifactorial variance analysis was performed by using as factors the method (chips and barrel) and duration of ageing (30,60, and 90 days). It was determined which factors had a statistically significant effect on basic chemical composition and on the amount of volatile compounds. Statistical tests were made using the SPSS 19.0 statistical analysis (IBM, Armonk, NY, USA) and a Tukey HSD (Honestly Significant Difference) test with a confidence interval of $95 \%$ or $99 \%$. Differences were considered to be significant at $p<0.05$. All analyses were done in triplicate, and the results are presented as the mean values and standard deviations. Partial least squares regression (PLSR) was carried out to separate wines through duration and method of ageing by the chemical concentration of volatile compounds from wood $(\mathrm{Y})$ with initial wine chemical concentrations of volatile compounds (X), using the XLSTAT 2020.3.1.1001 (Addinsoft, Paris, France).

\section{Results and Discussion}

In Romania, Muscat Ottonel variety is cultivated on the surface of 5547 ha (5.95\% from total vineyard surface) mainly in Transylvania (Central Romania), Moldavia (Eastern Romania), and Dobrogea (South-Eastern Romania) [22]. Lechinta vineyard is ideal for viticulture, due to brown soils and clay (illuvial), rich in calcium and iron. The climate is temperate-continental with hot and humid summers, followed by warm autumns; the vineyard is planted on Eastern and Western exposure at an altitude of 300-500 m high. The flavours are preserved and the high level of acidity is maintained due to the foggy days of late summer and early autumn in Lechinta vineyard during ripening. Muscat Ottonel wines are characterized by the presence of free and glycosylated monoterpenoids, and several aromatic compounds like fatty acids and phenolic compounds. The most important terpenes are linalool, geraniol, nerol, citronellol, and $\alpha$-terpineol, which are responsible for the characteristic floral notes of the wines [23]. However, the presence and concentration of these compounds in grapes and wines depend on several factors, like cultivar, climate, soil, agricultural practices, and winemaking process [24].

\subsection{Chemical Composition of Aged Wines}

The ageing of wine in oak wood barrels and oak chips is associated with changes in the oenological parameters of the maturing wine. The main chemical compound of wine, ethanol, is decreasing as a consequence of its preferential evaporation through the oak wood staves [25]. In our study, wine with an ethanol content of $12.49 \%$ vol.alc. was stored in closed demijohns with oak wood chips, and oak barrel, respectively. The result of the analysis made in successive ageing periods indicated that the ethanol content had decreased (Table 1). The ethanol loss ranged from 1.92 to $3.12 \%$ vol.alc, so statistically, this parameter was affected by ageing method (chips or barrel), as well as ageing period, but not by the types of chips (Table 1). 
Table 1. Oenological analyses of white wines aged with oak chips and barrel for 30, 60, and 90 days; multivariate analysis of variance (MANOVA) taking as factors time, types of oak chips and ageing method.

\begin{tabular}{|c|c|c|c|c|c|c|c|c|c|c|c|c|c|}
\hline \multirow{2}{*}{$\begin{array}{c}\text { Parameters } \\
\text { Days }\end{array}$} & \multirow{2}{*}{$\begin{array}{l}\text { Initial } \\
\text { Wine }\end{array}$} & \multicolumn{3}{|c|}{ Untoasted Oak Chips } & \multicolumn{3}{|c|}{ Light Toasted Chips } & \multicolumn{3}{|c|}{ Untoasted Barrel } & \multicolumn{3}{|c|}{ MANOVA } \\
\hline & & 30 & 60 & 90 & 30 & 60 & 90 & 30 & 60 & 90 & Time & $\begin{array}{l}\text { Chips } \\
\text { Type }\end{array}$ & $\begin{array}{l}\text { Ageing } \\
\text { Method }\end{array}$ \\
\hline Ethanol (\% ABV) & $\begin{array}{c}12.49 \pm \\
0.17^{\mathrm{a}}\end{array}$ & $\begin{array}{c}12.50 \pm \\
0.36^{\mathrm{a}}\end{array}$ & $\begin{array}{l}12.25 \pm \\
0.2^{\mathrm{b}}\end{array}$ & $\begin{array}{c}12.14^{ \pm} \\
0.05^{\mathrm{c}}\end{array}$ & $\begin{array}{c}12.51 \pm \\
0.35^{\mathrm{a}}\end{array}$ & $\begin{array}{c}12.25 \pm \\
0.2^{\mathrm{b}}\end{array}$ & $\begin{array}{c}12.14^{ \pm} \\
0.05^{\mathrm{c}}\end{array}$ & $\begin{array}{l}12.32 \pm \\
0.12 \mathrm{ab}\end{array}$ & $\begin{array}{r}12.17^{ \pm} \\
0.07^{\mathrm{c}}\end{array}$ & $\begin{array}{r}12.10^{ \pm} \\
0.05^{\mathrm{c}}\end{array}$ & $* * *$ & ns & $* *$ \\
\hline $\begin{array}{l}\text { Volatile acidity } \\
\text { (g/L acetic acid) }\end{array}$ & $\begin{array}{l}0.21 \pm \\
0.01 \mathrm{~d}\end{array}$ & $\begin{array}{l}0.21 \pm \\
0.01 \mathrm{~d}\end{array}$ & $\begin{array}{l}0.29 \pm \\
0.01^{\mathrm{c}}\end{array}$ & $\begin{array}{l}0.31 \pm \\
0.01 \mathrm{bc}\end{array}$ & $\begin{array}{l}0.21 \pm \\
0.02^{d}\end{array}$ & $\begin{array}{l}0.28 \pm \\
0.01^{\mathrm{c}}\end{array}$ & $\begin{array}{l}0.31 \pm \\
0.01 \mathrm{bc}\end{array}$ & $\begin{array}{l}0.29 \pm \\
0.02^{c}\end{array}$ & $\begin{array}{l}0.34 \pm \\
0.02^{b}\end{array}$ & $\begin{array}{l}0.39 \pm \\
0.02^{\mathrm{a}}\end{array}$ & $* * *$ & $* * *$ & $* *$ \\
\hline $\begin{array}{l}\text { Total acidity }(\mathrm{g} / \mathrm{L} \\
\text { tartaric acid) }\end{array}$ & $\begin{array}{l}4.67 \pm \\
0.03^{\mathrm{a}}\end{array}$ & $\begin{array}{l}4.62 \pm \\
0.03 \mathrm{ab}\end{array}$ & $\begin{array}{l}4.55 \pm \\
0.06 \mathrm{ab}\end{array}$ & $\begin{array}{l}4.52 \pm \\
0.05^{b}\end{array}$ & $\begin{array}{l}4.63 \pm \\
0.02 \mathrm{ab}\end{array}$ & $\begin{array}{l}4.55 \pm \\
0.06 \mathrm{ab}\end{array}$ & $\begin{array}{l}4.52 \pm \\
0.05^{b}\end{array}$ & $\begin{array}{l}4.63 \pm \\
0.02 \mathrm{ab}\end{array}$ & $\begin{array}{l}4.55 \pm \\
0.06^{\mathrm{ab}}\end{array}$ & $\begin{array}{l}4.52 \pm \\
0.05^{b}\end{array}$ & $* *$ & ns & ns \\
\hline Dry extract (g/L) & $\begin{array}{c}21.47 \pm \\
0.5^{\mathrm{a}}\end{array}$ & $\begin{array}{c}21.39 \pm \\
0.4^{\mathrm{a}}\end{array}$ & $\begin{array}{c}21.39 \pm \\
0.4^{\mathrm{a}}\end{array}$ & $\begin{array}{c}21.36 \pm \\
0.35^{\mathrm{a}}\end{array}$ & $\begin{array}{c}21.39 \pm \\
0.4^{\mathrm{a}}\end{array}$ & $\begin{array}{c}21.39 \pm \\
0.4^{\mathrm{a}}\end{array}$ & $\begin{array}{c}21.36 \pm \\
0.35^{\mathrm{a}}\end{array}$ & $\begin{array}{c}21.38 \pm \\
0.38^{a}\end{array}$ & $\begin{array}{l}21.4 \pm \\
0.39^{a}\end{array}$ & $\begin{array}{c}21.35 \pm \\
0.34^{\mathrm{a}}\end{array}$ & ns & ns & ns \\
\hline $\begin{array}{l}\text { Non-reducing dry } \\
\text { extract }(\mathrm{g} / \mathrm{L})\end{array}$ & $\begin{array}{c}20.07 \pm \\
0.06^{\mathrm{a}}\end{array}$ & $\begin{array}{c}20.06 \pm \\
0.05^{\mathrm{a}}\end{array}$ & $\begin{array}{c}20.05^{ \pm} \\
0.05^{\mathrm{a}}\end{array}$ & $\begin{array}{c}20.02 \pm \\
0.03^{\mathrm{a}}\end{array}$ & $\begin{array}{c}20.06 \pm \\
0.05^{\mathrm{a}}\end{array}$ & $\begin{array}{c}20.05^{ \pm} \\
0.03^{a}\end{array}$ & $\begin{array}{c}20.02 \pm \\
0.03^{\mathrm{a}}\end{array}$ & $\begin{array}{c}20.06 \pm \\
0.05^{\mathrm{a}}\end{array}$ & $\begin{array}{c}20.05 \pm \\
0.5^{\mathrm{a}}\end{array}$ & $\begin{array}{c}20.01 \pm \\
0.01^{\mathrm{a}}\end{array}$ & ns & ns & ns \\
\hline Free $\mathrm{SO}_{2}(\mathrm{mg} / \mathrm{L})$ & $\begin{array}{c}20.33 \pm \\
0.58^{\mathrm{a}}\end{array}$ & $\begin{array}{c}19.00 \pm \\
1.0^{\mathrm{b}}\end{array}$ & $\begin{array}{l}18.17 \pm \\
1.26^{\mathrm{ab}}\end{array}$ & $\begin{array}{l}17.33 \pm \\
1.53^{\mathrm{ab}}\end{array}$ & $\begin{array}{c}19.00 \pm \\
1.00^{\mathrm{a}}\end{array}$ & $\begin{array}{l}18.19 \pm \\
1.25^{\mathrm{ab}} \\
\end{array}$ & $\begin{array}{l}17.37 \pm \\
1.48^{\mathrm{ab}}\end{array}$ & $\begin{array}{l}18.33 \pm \\
0.58^{a b}\end{array}$ & $\begin{array}{l}17.00 \mathrm{a} \\
1.00 \mathrm{ab}\end{array}$ & $\begin{array}{c}15.33 \pm \\
1.53^{\mathrm{b}}\end{array}$ & * & $* *$ & $* *$ \\
\hline Total $\mathrm{SO}_{2}(\mathrm{mg} / \mathrm{L})$ & $\begin{array}{l}129 \pm \\
1.53^{\mathrm{cd}}\end{array}$ & $\begin{array}{l}132 \pm \\
1.73 \mathrm{bc}\end{array}$ & $\begin{array}{l}136 \pm \\
2.00^{\mathrm{ab}}\end{array}$ & $\begin{array}{l}139 \pm \\
1.00^{\mathrm{a}}\end{array}$ & $\begin{array}{l}136 \pm \\
3.00 \mathrm{bc}\end{array}$ & $\begin{array}{l}135 \pm \\
1.00 \mathrm{ab}\end{array}$ & $\begin{array}{l}139 \pm \\
1.00^{\mathrm{a}}\end{array}$ & $\begin{array}{l}125 \pm \\
1.00 \mathrm{de}\end{array}$ & $\begin{array}{l}122 \pm \\
2.00 \text { ef }\end{array}$ & $\begin{array}{l}119 \pm \\
1.00^{f}\end{array}$ & $* * *$ & $* * *$ & $* * *$ \\
\hline $\mathrm{pH}$ & $\begin{array}{l}3.50 \pm \\
0.02^{\mathrm{a}}\end{array}$ & $\begin{array}{l}3.50 \pm \\
0.01^{\mathrm{a}}\end{array}$ & $\begin{array}{l}3.50 \pm \\
0.03^{\mathrm{a}}\end{array}$ & $\begin{array}{l}3.51 \pm \\
0.02^{\mathrm{a}}\end{array}$ & $\begin{array}{l}3.51 \pm \\
0.02^{\mathrm{a}}\end{array}$ & $\begin{array}{l}3.52 \pm \\
0.01^{\mathrm{a}}\end{array}$ & $\begin{array}{l}3.51 \pm \\
0.02^{\mathrm{a}}\end{array}$ & $\begin{array}{c}3.52 \pm \\
0.01^{\mathrm{a}}\end{array}$ & $\begin{array}{l}3.52 \pm \\
0.02^{\mathrm{a}}\end{array}$ & $\begin{array}{c}3.53 \pm \\
0.03^{\mathrm{a}}\end{array}$ & ns & ns & ns \\
\hline
\end{tabular}

Average value \pm standard deviation $(n=3)$. Roman letters are significance of difference $(p \leq 0.05)$ between variants in the same row. The difference between any two values, followed by at

least one common letter, is insignificant. * $p \leq 0.05 ; * *: p \leq 0.01 ;{ }^{* * *}: p \leq 0.001 ;$ ns: not significant. 
Assessing the effect of ageing method on the ethanol content changes, the statistically significant differences were observed for barrel-aged variants. In aged wines, the loss of alcohol content could not be explained based on oxidation reactions of ethanol by film yeasts or by a coupled autoxidation of phenolics since the highest acetoin concentration was detected in treated wines (chips and barrel). The lower ethanol content for barrel-aged variants could be attributed to the penetration of ethanol into the porous structure of the oak wood during maturation [25]. Another important parameter of wines, total and volatile acidity presented different changes during ageing period. Wines treated with oak chips (untoasted and toasted) and aged in barrels presented significantly lower amounts of tartaric acid than initial wine. Total acidity was not influenced by ageing method and chips types. On the other hand, acetic acid content was significantly affected by method and ageing period, with a slight increase, after 90 days. As may be seen in Table 1, high volatile acidity in barrel-aged wines was detected compared with chips aged wines in demijohns, which may be due to microbiological factors (level of presence of bacteria). The extraction of phenolic and volatile carboxylic acids from wood could also increase in volatile acidity [12]. The total and volatile acidity were significantly dependent on ageing time. Volatile acidity was also significantly dependent on chips types and ageing method. The results showed that the levels of total $\mathrm{SO}_{2}$ were below the legal limits indicated by the International Organization of Vine and Wine. During ageing, occurred interesting changes of $\mathrm{SO}_{2}$ amounts, and showed an increased level in wines treated with chips and a slight decrease in barrel-aged wines compared with initial wine. The total $\mathrm{SO}_{2}$ losses during the barrel ageing process can be attributed to oxygen permeability through staves [26].

\subsection{Volatile Profile of Aged Wines}

The volatile compounds play an important role in the organoleptic characteristics of the wine. The flavour of the wine is influenced due to a large number of compounds present in it. These volatile compounds present in wine have been classified according to their chemical structure: alcohols, acids, lactones, aldehydes, ketones, and esters, as the main constituent, and important. These compounds have a different chemical nature with a wide range of polarity, volatility, solubility, and $\mathrm{pH}$ values; some of these volatile compounds can only be found at very low concentrations, and a part of them are unstable. Sample preparation, and especially the extraction method are important factors in the determination of these substances.

The liquid-liquid extraction and GC-MS analysis of white wines before ageing and after 30, 60, and 90 days of ageing in barrel and with oak chips identified seven chemical groups: alcohols (13), carbonyls (1), carboxylic acids (10), esters (16), lactones (3), terpenes (3), and volatile phenols (5) (Table 2). Multivariate analysis was used to determine the influence of factors such as ageing period, type of oak chips (untoasted and light toasted), and method of ageing (chips and barrel) on volatile compounds. Visible changes in volatile compounds correlated with ageing process were observed, suggesting that the aged wines had different profiles compared with initial wine. During ageing, specific compounds are transferred from the wood into wines as a result of the contact with the oak chips or barrel. Each sample of wine evolved in a different way, with fluctuations of the volatile compounds in wines aged for 30,60, and 90 days with either oak chips or oak barrel. Most of the volatile compounds are commonly found in wines and are derived from grapes and yeast strain fermentation and the vinification process [27]. Alcohols, esters, and carboxylic acids were the main compounds in wines samples. 
Table 2. Quantitative analysis of volatile compounds of white wines aged with oak chips and barrel. All values are expressed as means (mg/L and $\mu \mathrm{g} / \mathrm{L}) \pm \mathrm{standard}$ deviation (SD). Multivariate analysis of variance (MANOVA) taking as factors duration of ageing (30, 60, and 90 days), chips types (untoasted and light toasted), and ageing method. The results are expressed in equivalents of phenyl ethanol.

\begin{tabular}{|c|c|c|c|c|c|c|c|c|c|c|c|c|c|}
\hline Compounds & \multirow{2}{*}{$\begin{array}{l}\text { Initial } \\
\text { Wine }\end{array}$} & \multicolumn{3}{|c|}{ Untoasted Oak Chips } & \multicolumn{3}{|c|}{ Light Toasted Chips } & \multicolumn{3}{|c|}{ Untoasted Barrel } & \multicolumn{3}{|c|}{ MANOVA } \\
\hline Duration of Ageing (Days) & & 30 & 60 & 90 & 30 & 60 & 90 & 30 & 60 & 90 & Time & $\begin{array}{l}\text { Chips } \\
\text { Type }\end{array}$ & $\begin{array}{l}\text { Ageing } \\
\text { Methoo }\end{array}$ \\
\hline \multicolumn{14}{|c|}{ Alcohols } \\
\hline \multicolumn{14}{|c|}{ Major alcohols (mg/L) } \\
\hline Isobutanol & $48 \pm 0.5^{\mathrm{a}}$ & $39 \pm 1.9^{\mathrm{de}}$ & $41 \pm 0.5^{\text {cde }}$ & $42 \pm 0.6^{\mathrm{bcd}}$ & $40 \pm 1.3^{\text {cde }}$ & $43 \pm 1.2^{b c}$ & $44 \pm 1.6^{\mathrm{ab}}$ & $39 \pm 1.7$ de & $40 \pm 1.0^{\text {cde }}$ & $42 \pm 1.2^{b c d}$ & * & * & ns \\
\hline Isoamyl alcohol & $55 \pm 0.1^{\mathrm{ab}}$ & $55 \pm 0.8^{\mathrm{ab}}$ & $53 \pm 0.9^{a b}$ & $55 \pm 0.8^{\mathrm{ab}}$ & $53 \pm 2.7^{a b}$ & $54 \pm 1.8^{a b}$ & $55 \pm 1.7^{\mathrm{ab}}$ & $49 \pm 2.7^{b}$ & $52 \pm 2.4^{\mathrm{ab}}$ & $53 \pm 3.0^{\mathrm{ab}}$ & ns & ns & ns \\
\hline 2-phenylethanol & $22 \pm 1.3^{a}$ & $20 \pm 0.9^{a}$ & $19 \pm 0.9^{\mathrm{a}}$ & $19 \pm 0.9^{\mathrm{a}}$ & $19 \pm 0.9^{\mathrm{a}}$ & $19 \pm 0.9^{\mathrm{a}}$ & $19 \pm 1.8^{\mathrm{a}}$ & $19 \pm 1.8^{\mathrm{a}}$ & $20 \pm 1.1^{\mathrm{a}}$ & $19 \pm 1.0^{\mathrm{a}}$ & ns & ns & ns \\
\hline \multicolumn{14}{|c|}{ Minor alcohols $(\mu \mathrm{g} / \mathrm{L})$} \\
\hline Hexanol & $2570 \pm 19^{c}$ & $2014 \pm 11^{\mathrm{e}}$ & $2745 \pm 20^{b}$ & $3109 \pm 8.7^{\mathrm{a}}$ & $2348 \pm 49^{d}$ & $2565 \pm 60^{c}$ & $2535 \pm 60^{c}$ & $2382 \pm 26^{d}$ & $2613 \pm 43^{c}$ & $3123 \pm 26^{\mathrm{a}}$ & $* * *$ & $* *$ & $* *$ \\
\hline 4-methyl-1-pentanol & $32 \pm 1.7^{c}$ & $44 \pm 2.3^{\mathrm{ab}}$ & $41 \pm 0.0^{\mathrm{b}}$ & $31 \pm 0.6^{c}$ & $30 \pm 1.4^{\mathrm{cd}}$ & $48 \pm 4.3^{\mathrm{a}}$ & $21 \pm 1.5^{\mathrm{e}}$ & $42 \pm 2.3^{a b}$ & $28 \pm 3.9^{\mathrm{cd}}$ & $24 \pm 1.6^{\mathrm{e}}$ & $* *$ & ** & ** \\
\hline E-3-hexenol & $17 \pm 1.3^{\mathrm{bcd}}$ & $16 \pm 2.7^{\mathrm{cd}}$ & $19 \pm 2.3^{\mathrm{abcd}}$ & $14 \pm 1.9^{\mathrm{d}}$ & $15 \pm 1.2^{\mathrm{d}}$ & $22 \pm 1.9^{\mathrm{abc}}$ & $22 \pm 1.4^{\mathrm{abc}}$ & $17 \pm 2.2^{\mathrm{bcd}}$ & $14 \pm 1.9^{\mathrm{d}}$ & $23 \pm 0.9^{a}$ & $*$ & $*$ & $*$ \\
\hline Z-3-hexenol & $148 \pm 4.8^{\mathrm{c}}$ & $188 \pm 8.3^{\mathrm{ab}}$ & $181 \pm 9.0^{\mathrm{b}}$ & $130 \pm 10.0^{c}$ & $127 \pm 4.8^{c}$ & $205 \pm 6.3^{a}$ & $139 \pm 4.0^{c}$ & $182 \pm 10.7^{\mathrm{ab}}$ & $137 \pm 10.5^{c}$ & $132 \pm 10.2^{c}$ & * & $*$ & $*$ \\
\hline 2-nonanol & $87 \pm 3.9^{b}$ & $77 \pm 6.3^{b c}$ & $79 \pm 5.4^{b c}$ & $46 \pm 4.3^{\mathrm{e}}$ & $76 \pm 1.2^{c}$ & $61 \pm 0.3^{\mathrm{d}}$ & $55 \pm 1.7$ de & $92 \pm 1.8^{\mathrm{a}}$ & $64 \pm 1.9^{\mathrm{d}}$ & $49 \pm 4.6^{\mathrm{e}}$ & ** & ** & ** \\
\hline 1-heptanol & $175 \pm 4.2^{\mathrm{a}}$ & $159 \pm 8.4^{\mathrm{b}}$ & $172 \pm 1.4^{\mathrm{ab}}$ & $171 \pm 85^{\mathrm{ab}}$ & $163 \pm 3.1^{\mathrm{ab}}$ & $176 \pm 3.1^{\mathrm{a}}$ & $168 \pm 3.5^{\mathrm{ab}}$ & $165 \pm 4.75^{\mathrm{ab}}$ & $162 \pm 2.8^{b}$ & $170 \pm 3.0^{\mathrm{ab}}$ & * & ns & ns \\
\hline 2,3-butanediol & $622 \pm 10^{f}$ & $625 \pm 19^{f}$ & $1510 \pm 17^{\mathrm{b}}$ & $1395 \pm 30^{c}$ & $514 \pm 13 \mathrm{~g}$ & $1544 \pm 40^{\mathrm{b}}$ & $1886 \pm 40^{\mathrm{a}}$ & $646 \pm 10^{f}$ & $926 \pm 13^{e}$ & $1291 \pm 18^{\mathrm{d}}$ & $* * *$ & $* * *$ & $* * *$ \\
\hline 3-methylthio-1-propanol & $836 \pm 6^{a}$ & $689 \pm 8.8^{c}$ & $780 \pm 19^{b}$ & $515 \pm 14^{\mathrm{e}}$ & $478 \pm 19^{\mathrm{f}}$ & $654 \pm 3.9^{d}$ & $363 \pm 3.0^{g}$ & $471 \pm 9.4^{\mathrm{f}}$ & $647 \pm 15^{\mathrm{d}}$ & $483 \pm 6.5^{\text {ef }}$ & $* *$ & ** & ** \\
\hline Glycerol & $20 \pm 0.9^{f}$ & $114 \pm 10^{\mathrm{e}}$ & $184 \pm 12^{c}$ & $280 \pm 9.8^{b}$ & $106 \pm 5.8^{\mathrm{e}}$ & $125 \pm 2.0^{\mathrm{e}}$ & $303 \pm 10^{b}$ & $157 \pm 6.8^{\mathrm{d}}$ & $179 \pm 4.1^{\mathrm{cd}}$ & $315 \pm 8.0^{\mathrm{a}}$ & $* * *$ & $* *$ & $* *$ \\
\hline Benzyl alcohol & $48 \pm 1.7^{\mathrm{a}}$ & $41 \pm 2.2^{b c}$ & $31 \pm 1.7^{\mathrm{d}}$ & $37 \pm 1.9^{\mathrm{bcd}}$ & $42 \pm 2^{\mathrm{ab}}$ & $34 \pm 3^{\mathrm{cd}}$ & $37 \pm 4.4^{\mathrm{bcd}}$ & $43 \pm 0.9^{\mathrm{ab}}$ & $35 \pm 2.7^{\mathrm{cd}}$ & $37 \pm 0.6^{\mathrm{bcd}}$ & * & $*$ & $*$ \\
\hline \multicolumn{14}{|c|}{ Terpenes $(\mu \mathrm{g} / \mathrm{L})$} \\
\hline Linalool & $1027 \pm 19^{b}$ & $1053 \pm 20^{b}$ & $913 \pm 10^{\mathrm{d}}$ & $725 \pm 20 \mathrm{~g}$ & $792 \pm 9.5^{\mathrm{f}}$ & $674 \pm 17^{\mathrm{h}}$ & $491 \pm 10^{\mathrm{i}}$ & $1084 \pm 21^{a}$ & $974 \pm 10^{c}$ & $846 \pm 12^{\mathrm{e}}$ & $* * *$ & $* * *$ & $* * *$ \\
\hline Terpineol & $1180 \pm 13^{\mathrm{a}}$ & $1090 \pm 7.1^{b}$ & $975 \pm 6.8^{c}$ & $915 \pm 7.0^{d}$ & $979 \pm 34^{c}$ & $919 \pm 15^{d}$ & $905 \pm 9.7^{\mathrm{d}}$ & $971 \pm 9.9^{c}$ & $980 \pm 9.5^{c}$ & $949 \pm 4.4^{\mathrm{cd}}$ & $* *$ & * & $*$ \\
\hline Trans-geraniol & $700 \pm 19^{\mathrm{a}}$ & $680 \pm 20^{a}$ & $590 \pm 9.6^{c}$ & $470 \pm 10^{\mathrm{e}}$ & $480 \pm 9.8 \mathrm{de}$ & $510 \pm 10^{\mathrm{d}}$ & $315 \pm 4.8^{\mathrm{g}}$ & $630 \pm 5.0^{b}$ & $390 \pm 9.8^{f}$ & $234 \pm 2.4^{\mathrm{h}}$ & $* * *$ & $* * *$ & $* * *$ \\
\hline \multicolumn{14}{|c|}{ Major carbonyls (mg/L) } \\
\hline Acetoin & $72 \pm 2 \mathrm{~g}$ & $91 \pm 2.2$ def & $106 \pm 5.4^{\mathrm{d}}$ & $151 \pm 10^{b}$ & $87 \pm 4.2^{\text {efg }}$ & $102 \pm 2.0^{\mathrm{de}}$ & $130 \pm 9.9^{c}$ & $84 \pm 1.9^{\mathrm{fg}}$ & $127 \pm 9.9^{c}$ & $197 \pm 2.84^{\mathrm{a}}$ & $* * *$ & ** & ** \\
\hline
\end{tabular}


Table 2. Cont

\begin{tabular}{|c|c|c|c|c|c|c|c|c|c|c|c|c|c|}
\hline Compounds & \multirow{2}{*}{$\begin{array}{l}\text { Initial } \\
\text { Wine }\end{array}$} & \multicolumn{3}{|c|}{ Untoasted Oak Chips } & \multicolumn{3}{|c|}{ Light Toasted Chips } & \multicolumn{3}{|c|}{ Untoasted Barrel } & \multicolumn{3}{|c|}{ MANOVA } \\
\hline Duration of Ageing (Days) & & 30 & 60 & 90 & 30 & 60 & 90 & 30 & 60 & 90 & Time & $\begin{array}{l}\text { Chips } \\
\text { Type }\end{array}$ & $\begin{array}{l}\text { Ageing } \\
\text { Method }\end{array}$ \\
\hline \multicolumn{14}{|c|}{ Carboxylic acids $(\mu \mathrm{g} / \mathrm{L})$} \\
\hline Butanoic acid & $23 \pm 2.1 \mathrm{e}$ & $65 \pm 3.8^{b}$ & $88 \pm 5^{\mathrm{a}}$ & $41 \pm 0.9^{\mathrm{d}}$ & $51 \pm 1.6^{c}$ & $91 \pm 5.6^{\mathrm{a}}$ & $47 \pm 2^{d}$ & $67 \pm 2.4^{\mathrm{b}}$ & $72 \pm 1.3^{b}$ & $42 \pm 2.9^{d}$ & $* * *$ & $* *$ & $* *$ \\
\hline Hexanoic acid & $12 \pm 1.2^{\mathrm{f}}$ & $28 \pm 0.2^{\mathrm{e}}$ & $38 \pm 0.6^{\mathrm{d}}$ & $47 \pm 0.9^{c}$ & $37 \pm 1.3^{\mathrm{d}}$ & $57 \pm 0.4^{\mathrm{b}}$ & $66 \pm 0.1^{\mathrm{a}}$ & $29 \pm 0.8^{\mathrm{e}}$ & $37 \pm 1.0^{\mathrm{d}}$ & $47 \pm 1^{\mathrm{c}}$ & $* * *$ & ** & ** \\
\hline Isovaleric acid & $68 \pm 8.8^{\mathrm{e}}$ & $150 \pm 9.16^{\mathrm{bc}}$ & $175 \pm 7.9^{\mathrm{a}}$ & $140 \pm 2.2^{\mathrm{cd}}$ & $124 \pm 0.9^{\mathrm{d}}$ & $163 \pm 9.25^{\mathrm{ab}}$ & $142 \pm 1.2^{\mathrm{cd}}$ & $156 \pm 9.5^{a b c}$ & $166 \pm 1.4^{\mathrm{ab}}$ & $140 \pm 2.2^{\mathrm{cd}}$ & $* * *$ & ** & $*$ \\
\hline Lactic acid & $130 \pm 2.8^{\mathrm{f}}$ & $155 \pm 4^{\mathrm{e}}$ & $162 \pm 2^{\mathrm{e}}$ & $376 \pm 3.0^{\mathrm{b}}$ & $165 \pm 3.3^{\mathrm{e}}$ & $193 \pm 2.9^{\mathrm{d}}$ & $349 \pm 4.7^{c}$ & $161 \pm 8.3^{\mathrm{e}}$ & $375 \pm 7.3^{b}$ & $548 \pm 8.7^{\mathrm{a}}$ & $* * *$ & $* *$ & * \\
\hline Octanoic acid & $3267 \pm 12^{f}$ & $3270 \pm 12^{f}$ & $3469 \pm 36^{d}$ & $3406 \pm 15^{\mathrm{e}}$ & $3525 \pm 21^{c}$ & $3408 \pm 17^{\mathrm{e}}$ & $3518 \pm 15^{c}$ & $3566 \pm 14^{c}$ & $3979 \pm 16^{a}$ & $3626 \pm 19^{b}$ & $* * *$ & $* *$ & $* *$ \\
\hline Decanoic acid & $1588 \pm 17^{a}$ & $978 \pm 22^{b}$ & $574 \pm 23$ de & $608 \pm 7.5^{\mathrm{d}}$ & $587 \pm 20$ de & $518 \pm 8.9^{\mathrm{f}}$ & $460 \pm 9.7^{g}$ & $690 \pm 95^{c}$ & $589 \pm 8.9$ de & $562 \pm 3.0^{\mathrm{e}}$ & $* * *$ & $* *$ & ** \\
\hline E-2-hexenoic acid & $10 \pm 0.8^{\mathrm{e}}$ & $49 \pm 3.2^{\mathrm{a}}$ & $33 \pm 4.7^{c}$ & $34 \pm 2.1^{c}$ & $15 \pm 1.7 \mathrm{de}$ & $16 \pm 0.2^{\mathrm{de}}$ & $16 \pm 0.8^{\mathrm{de}}$ & $43 \pm 0.4^{\mathrm{ab}}$ & $38 \pm 0.8^{b}$ & $21 \pm 0.4^{\mathrm{d}}$ & $* *$ & $* *$ & $* *$ \\
\hline Hexadecanoic acid & $97 \pm 1.5^{\mathrm{a}}$ & $22 \pm 2.4^{\mathrm{e}}$ & $33 \pm 0.8^{\mathrm{d}}$ & $41 \pm 0.8^{b}$ & $9.8 \pm 2.1^{f}$ & $23 \pm 0.8^{\mathrm{e}}$ & $32 \pm 1.7^{\mathrm{d}}$ & $12 \pm 1.3^{f}$ & $36 \pm 2.3^{\mathrm{cd}}$ & $41 \pm 1.2^{b}$ & $* * *$ & $* * *$ & $* *$ \\
\hline Pydolic acid & $93 \pm 2.3^{a}$ & $68 \pm 0.9^{b c}$ & $63 \pm 0.9^{\mathrm{cd}}$ & $59 \pm 1.7^{\mathrm{d}}$ & $72 \pm 3.4^{b}$ & $59 \pm 3.8^{\mathrm{d}}$ & $49 \pm 2.6^{\mathrm{e}}$ & $66 \pm 4^{\mathrm{bcd}}$ & $66 \pm 1.1 \mathrm{bcd}$ & $59 \pm 3.8^{\mathrm{d}}$ & $* *$ & ${ }^{*}$ & $*$ \\
\hline 2-oxoapidic & $7 \pm 0.4^{\mathrm{d}}$ & $20 \pm 1.9^{b}$ & $21 \pm 1.4^{\mathrm{ab}}$ & $15 \pm 1.7^{c}$ & $21 \pm 1.1^{\mathrm{ab}}$ & $25 \pm 1.4^{\mathrm{a}}$ & $25 \pm 0.9^{\mathrm{a}}$ & $22 \pm 0.9 \mathrm{ab}$ & $21 \pm 0.6^{\mathrm{ab}}$ & $21 \pm 0.5^{\mathrm{ab}}$ & $*$ & ns & ns \\
\hline \multicolumn{14}{|c|}{ Esters $(\mu \mathrm{g} / \mathrm{L})$} \\
\hline Isoamyl acetate & $1218 \pm 3.4^{\mathrm{d}}$ & $1968 \pm 28^{c}$ & $2235 \pm 62^{b}$ & $2497 \pm 79^{\mathrm{a}}$ & $1990 \pm 17^{c}$ & $2308 \pm 79^{b}$ & $2512 \pm 87^{a}$ & $1955 \pm 9.8^{c}$ & $1975 \pm 8.8^{c}$ & $2183 \pm 4.6^{b}$ & $* * *$ & ** & ** \\
\hline Hexyl acetate & $112 \pm 1^{\mathrm{b}}$ & $92 \pm 3.7^{c}$ & $75 \pm 1.4^{\mathrm{de}}$ & $66 \pm 1.3^{f}$ & $122 \pm 0.5^{\mathrm{a}}$ & $107 \pm 4.5^{\mathrm{b}}$ & $88 \pm 1.0^{c}$ & $96 \pm 3.5^{c}$ & $77 \pm 5.9^{d}$ & $68 \pm 0.8^{\text {ef }}$ & $* * *$ & ** & $* *$ \\
\hline Ethyl hexanoate & $191 \pm 4.0^{\mathrm{bc}}$ & $210 \pm 10^{\mathrm{ab}}$ & $190 \pm 4.0^{b c}$ & $175 \pm 11.4^{c}$ & $148 \pm 6.5^{\mathrm{d}}$ & $216 \pm 6.7^{\mathrm{a}}$ & $113 \pm 2.4^{\mathrm{e}}$ & $195 \pm 9.8^{\mathrm{abc}}$ & $128 \pm 2.7^{\mathrm{de}}$ & $114 \pm 34^{\mathrm{e}}$ & $* *$ & ${ }^{*}$ & $*$ \\
\hline Ethyl lactate & $588 \pm 12 \mathrm{~g}$ & $1664 \pm 57^{\mathrm{d}}$ & $2178 \pm 96^{a}$ & $1918 \pm 91^{c}$ & $1229 \pm 73^{f}$ & $2104 \pm 89^{b}$ & $1352 \pm 46^{\mathrm{f}}$ & $1387 \pm 44^{\mathrm{f}}$ & $1506 \pm 94^{\mathrm{e}}$ & $1897 \pm 48^{c}$ & $* * *$ & $* * *$ & $* *$ \\
\hline Ethyl octanoate & $313 \pm 24^{\mathrm{h}}$ & $365 \pm 36^{\text {fgh }}$ & $485 \pm 10^{\mathrm{fg}}$ & $560 \pm 35^{\mathrm{ab}}$ & $385 \pm 14 \mathrm{fg}$ & $412 \pm 9.8^{\text {ef }}$ & $588 \pm 13^{\mathrm{a}}$ & $354 \pm 16^{\text {gh }}$ & $461 \pm 9$ de & $520 \pm 3.2 \mathrm{bc}$ & $* * *$ & $* *$ & $* *$ \\
\hline Ethyl 3-hydroxybutanoate & $74 \pm 4.1^{\mathrm{e}}$ & $428 \pm 27^{a b}$ & $476 \pm 4.5^{\mathrm{a}}$ & $452 \pm 3.3^{\mathrm{a}}$ & $252 \pm 7.1^{\mathrm{d}}$ & $367 \pm 3.9^{c}$ & $456 \pm 9.6^{\mathrm{a}}$ & $393 \pm 41$ bc & $467 \pm 14^{\mathrm{a}}$ & $432 \pm 10^{\mathrm{a}}$ & $*$ & $* *$ & $* *$ \\
\hline Ethyl decanoate & $205 \pm 7^{f}$ & $384 \pm 8.9^{d}$ & $447 \pm 3.0^{c}$ & $652 \pm 6.5^{\mathrm{a}}$ & $348 \pm 2.1^{\mathrm{e}}$ & $442 \pm 2.6^{c}$ & $534 \pm 0.3^{b}$ & $344 \pm 17^{\mathrm{e}}$ & $433 \pm 19^{c}$ & $523 \pm 19^{b}$ & $* * *$ & * & ** \\
\hline Monoethyl succinate & $2048 \pm 52^{c}$ & $2274 \pm 24^{b}$ & $2486 \pm 52^{a}$ & $2229 \pm 20^{c}$ & $1707 \pm 90^{\mathrm{f}}$ & $1846 \pm 69^{f}$ & $1907 \pm 99^{\mathrm{e}}$ & $1874 \pm 50^{\mathrm{def}}$ & $1488 \pm 94 \mathrm{~g}$ & $1401 \pm 22 \mathrm{~g}$ & $* * *$ & $* * *$ & $* * *$ \\
\hline Phenethyl acetate & $288 \pm 3^{f}$ & $304 \pm 0.5^{\mathrm{f}}$ & $539 \pm 2.7^{c}$ & $730 \pm 3.0^{\mathrm{a}}$ & $394 \pm 17^{\mathrm{e}}$ & $333 \pm 55$ ef & $402 \pm 21^{\mathrm{d}}$ & $305 \pm 25^{\mathrm{f}}$ & $526 \pm 22^{c}$ & $623 \pm 19^{b}$ & $* * *$ & $* * *$ & $* *$ \\
\hline Diethyl malate & $33 \pm 2^{f}$ & $62 \pm 8^{\mathrm{bcd}}$ & $53 \pm 3.2^{\text {cde }}$ & $64 \pm 5.6^{\mathrm{abc}}$ & $51 \pm 5^{\mathrm{de}}$ & $43 \pm 2.7^{\text {ef }}$ & $66 \pm 4.0^{\mathrm{ab}}$ & $62 \pm 3.3^{\mathrm{bcd}}$ & $76 \pm 3.2^{\mathrm{a}}$ & $74 \pm 1.9^{a b}$ & $* *$ & $* *$ & $* *$ \\
\hline Diethyl succinate & $116 \pm 12^{g}$ & $239 \pm 8^{\mathrm{cd}}$ & $226 \pm 5^{\mathrm{cd}}$ & $323 \pm 9.2^{\mathrm{a}}$ & $179 \pm 14^{\text {ef }}$ & $225 \pm 13^{\mathrm{cd}}$ & $151 \pm 15 \mathrm{fg}$ & $201 \pm 19$ de & $282 \pm 14^{\mathrm{b}}$ & $242 \pm 16^{c}$ & $* *$ & $* *$ & $* *$ \\
\hline $\begin{array}{c}\text { Diethyl } \\
\text { 2-hydroxy-3-methylsuccinate }\end{array}$ & $35 \pm 0.6^{f}$ & $42 \pm 3.7^{\text {ef }}$ & $43 \pm 3.4^{\text {ef }}$ & $57 \pm 1.8^{c}$ & $56 \pm 7.0^{c}$ & $89 \pm 3.9^{b}$ & $135 \pm 11^{\mathrm{a}}$ & $46 \pm 3.72$ cde & $48 \pm 1.1^{\text {cde }}$ & $55 \pm 1.6^{\mathrm{cd}}$ & $* *$ & * & * \\
\hline Trimethylene acetate & $486 \pm 14^{\mathrm{a}}$ & $361 \pm 39 \mathrm{bc}$ & $381 \pm 23^{b}$ & $274 \pm 18$ de & $238 \pm 15^{\mathrm{e}}$ & $307 \pm 10^{\mathrm{cd}}$ & $130 \pm 8.7^{\mathrm{f}}$ & $340 \pm 15^{b c}$ & $283 \pm 17$ de & $236 \pm 15^{\text {de }}$ & ** & ** & ** \\
\hline Ethyl glycinate & $30 \pm 1.9^{\mathrm{a}}$ & $19 \pm 0.5^{c}$ & $20 \pm 1.5^{\mathrm{bc}}$ & $23 \pm 0.4^{\mathrm{bc}}$ & $12 \pm 0.2^{\mathrm{de}}$ & $13 \pm 0.5^{\text {de }}$ & $16 \pm 0.6^{\mathrm{d}}$ & $24 \pm 2.5^{b}$ & $11 \pm 1.9^{\mathrm{e}}$ & $15 \pm 1.4^{\mathrm{d}}$ & $* *$ & $* *$ & $* *$ \\
\hline Citronellol acetate & $36 \pm 6.9 \mathrm{abc}$ & $29 \pm 4.9^{\mathrm{cd}}$ & $43 \pm 0.9 \mathrm{ab}$ & $21 \pm 1.7$ de & $44 \pm 2.8^{\mathrm{a}}$ & $22 \pm 1$ de & $23 \pm 0.7$ de & $34 \pm 3.7^{b c}$ & $24 \pm 3.5$ de & $19 \pm 1.3^{\mathrm{e}}$ & $* * *$ & ** & $* *$ \\
\hline Ethyl-4-hydroxybutanoate & $401 \pm 9.7^{\mathrm{a}}$ & $201 \pm 11^{c}$ & $278 \pm 8.9^{b}$ & $130 \pm 10^{\mathrm{e}}$ & $155 \pm 12$ de & $189 \pm 10^{c}$ & $181 \pm 8.2^{\mathrm{cd}}$ & $271 \pm 8.5^{b}$ & $201 \pm 12^{c}$ & $126 \pm 5.2^{\mathrm{e}}$ & $* * *$ & $* *$ & $* *$ \\
\hline
\end{tabular}


Table 2. Cont

\begin{tabular}{|c|c|c|c|c|c|c|c|c|c|c|c|c|c|}
\hline Compounds & \multirow{2}{*}{$\begin{array}{l}\text { Initial } \\
\text { Wine }\end{array}$} & \multicolumn{3}{|c|}{ Untoasted Oak Chips } & \multicolumn{3}{|c|}{ Light Toasted Chips } & \multicolumn{3}{|c|}{ Untoasted Barrel } & \multicolumn{3}{|c|}{ MANOVA } \\
\hline Duration of Ageing (Days) & & 30 & 60 & 90 & 30 & 60 & 90 & 30 & 60 & 90 & Time & $\begin{array}{l}\text { Chips } \\
\text { Type }\end{array}$ & $\begin{array}{l}\text { Ageing } \\
\text { Method }\end{array}$ \\
\hline \multicolumn{14}{|c|}{ Lactones $(\mu \mathrm{g} / \mathrm{L})$} \\
\hline Butyrolactone & $82 \pm 2.6^{g}$ & $285 \pm 10^{c}$ & $310 \pm 5.2^{b}$ & $320 \pm 5.8^{b}$ & $185 \pm 2.5^{\mathrm{f}}$ & $198 \pm 4.0^{\mathrm{f}}$ & $217 \pm 3.9^{\mathrm{e}}$ & $253 \pm 5.3^{d}$ & $287 \pm 3.4^{c}$ & $349 \pm 3.2^{\mathrm{a}}$ & $* * *$ & $* * *$ & $* * *$ \\
\hline Pantolactone & $19 \pm 0.9^{\mathrm{cd}}$ & $18 \pm 0.9^{\mathrm{cd}}$ & $20 \pm 0.9 \mathrm{bc}$ & $19 \pm 0.3^{\mathrm{cd}}$ & $29 \pm 1.1^{\mathrm{a}}$ & $22 \pm 1.2^{b}$ & $19 \pm 0.3^{\mathrm{cd}}$ & $20 \pm 0.8^{b c}$ & $19 \pm 0.2^{\mathrm{cd}}$ & $19 \pm 0.5^{\mathrm{cd}}$ & $* * *$ & * & ns \\
\hline 3,4-dimethyl-2(5)-furanone & $116 \pm 1.1^{\mathrm{a}}$ & $98 \pm 2.2^{c}$ & $103 \pm 2.2^{b}$ & $63 \pm 0.6^{\mathrm{e}}$ & $63 \pm 2.1^{\mathrm{e}}$ & $105 \pm 4.5^{b}$ & $94 \pm 5.1^{c}$ & $103 \pm 3.3^{b}$ & $76 \pm 3.0^{d}$ & $76 \pm 1.0^{\mathrm{d}}$ & $* * *$ & ** & $* *$ \\
\hline \multicolumn{14}{|c|}{ Volatile phenols ( $\mu \mathrm{g} / \mathrm{L})$} \\
\hline p-vinyl guaiacol & $25 \pm 0.8^{h}$ & $65 \pm 2.8^{g}$ & $89 \pm 2.0^{\mathrm{e}}$ & $105 \pm 3.0^{\mathrm{d}}$ & $126 \pm 5.1^{c}$ & $144 \pm 3.6^{b}$ & $156 \pm 2.5^{a}$ & $65 \pm 2.1 \mathrm{~g}$ & $79 \pm 3.6^{f}$ & $87 \pm 1.0^{\mathrm{ef}}$ & $* * *$ & $* * *$ & $* * *$ \\
\hline Methyl-hydroxycinnamate & $73 \pm 1.0^{\mathrm{a}}$ & $23 \pm 0.9^{\mathrm{e}}$ & $53 \pm 1.1$ bc & $13 \pm 1.1 \mathrm{~g}$ & $50 \pm 9.3^{c}$ & $58 \pm 4.9^{b}$ & $22 \pm 0.9^{\text {ef }}$ & $30 \pm 0.9^{\mathrm{d}}$ & $15 \pm 0.9^{\mathrm{fg}}$ & $12 \pm 0.4^{\mathrm{g}}$ & $* * *$ & $* * *$ & $* *$ \\
\hline Acetovanillone & $47 \pm 1.1^{\mathrm{h}}$ & $181 \pm 0.9^{b}$ & $164 \pm 4.8^{c}$ & $137 \pm 0.4^{\mathrm{de}}$ & $190 \pm 1.6^{\mathrm{a}}$ & $142 \pm 1.8^{\mathrm{d}}$ & $131 \pm 1.8^{\mathrm{f}}$ & $132 \pm 1.9$ ef & $126 \pm 1.7^{\mathrm{fg}}$ & $122 \pm 1 \mathrm{~g}$ & $* * *$ & $* * *$ & ** \\
\hline 2,3-hydroxybenzofurane & $411 \pm 10^{a}$ & $235 \pm 9.1^{c}$ & $148 \pm 7.1^{\text {de }}$ & $156 \pm 5.4^{\mathrm{d}}$ & $148 \pm 3.4^{\mathrm{de}}$ & $122 \pm 1.1^{\mathrm{fg}}$ & $115 \pm 2.2^{g}$ & $263 \pm 2.6^{b}$ & $142 \pm 1.7$ de & $135 \pm 1.9$ ef & $* * *$ & $* * *$ & ** \\
\hline Vanillin & $20 \pm 0.6^{c}$ & $124 \pm 0.6^{\mathrm{a}}$ & $123 \pm 0.3^{\mathrm{ab}}$ & $122 \pm 0.3^{b}$ & $124 \pm 0.9^{a}$ & $124 \pm 0.2^{a}$ & $123 \pm 0.5^{\mathrm{ab}}$ & $123 \pm 0.4^{\mathrm{ab}}$ & $123 \pm 0.8^{\mathrm{ab}}$ & $122 \pm 1^{b}$ & ** & ns & ns \\
\hline
\end{tabular}

Average value \pm standard deviation $(n=3)$. Roman letters are significance of difference $(p \leq 0.05)$ between variants in the same row. The difference between any two values, followed by at least one common letter, is insignificant. *: $p \leq 0.05 ; * *: p \leq 0.01 ; * * *: p \leq 0.001 ;$ ns: not significant. 


\subsubsection{Alcohols}

Alcohols were quantitatively the group with the highest concentration between volatile compounds. Higher alcohols have an important impact on the fruity aroma of wine [28] and they are the product of the yeast fermentation of sugars and the yeast metabolism of amino acids, carbohydrates, and lipids [29]. By using the LLE/GC-MS analysis, were detected 13 alcohols, consisting of 3 major alcohols (isoamyl alcohol, 2-phenylethanol, and isobutanol) and 10 minor alcohols (hexanol, 4-methyl-1-pentanol, E-3-hexenol, Z-3-hexenol, 2-nonanol, 1-heptanol, 2,3-butanediol, 3-methylthio-1-propanol, glycerol, and benzyl alcohol) (Table 2). As expected, the volatile compounds were detected at levels ranging from $\mathrm{ng} / \mathrm{L}$ to $\mathrm{mg} / \mathrm{L}$.

Between the major alcohols, isoamyl alcohol was the most abundant, with the concentrations of $55 \mathrm{mg} / \mathrm{L}$ in initial wine. This alcohol gives a cheesy note to the wine, and have an odour threshold of $30 \mathrm{mg} / \mathrm{L}$ [30], so in this case, may contribute to the aromatic profile. After 30 days of ageing, the concentration of this alcohol dropped to $54 \mathrm{mg} / \mathrm{L}$ (untoasted chips), $53 \mathrm{mg} / \mathrm{L}$ (light toasted chips) and $49 \mathrm{mg} / \mathrm{L}$ (untoasted barrels). At the end of ageing period (90 days), the amount of isoamyl alcohol slightly increased. The 2-phenylethanol with flowery, pollen, and perfumed notes [30] was found in $22 \mathrm{mg} / \mathrm{L}$ content in the initial wine. After 30 days ageing, the content in this compound decreased. The dynamic of this compound during ageing was interesting, with a continuous decrease in the variant with untoasted chips, and an increase in the variant with light toasted chips. During barrel ageing, 2-phenylthanol increased after 60 days, followed by a decrease after 90 days. As previously analysed alcohols, isobutanol (fusel, alcohol note) decreased after first 30 days of ageing, but begin to increase till the end of ageing to similar amount in the variants with untoasted oak chips, barrel ageing, and light toasted chips, respectively. The type of oak chips and ageing method did not significantly influence isoamyl alcohol, iso-butanol, and 2-phenylethanol concentration. Alcohols with minor abundance: 1-heptanol, E-3-hexenol, Z-3-hexenol, and 2-nonanol bring predominantly green aroma tones, while higher saturated alcohols, like benzyl alcohol, are responsible for citrus-like smells [30]. The changes of minor alcohols observed during ageing may be set in three trends. In Trend I, the concentrations of alcohols like 2-nonanol and benzyl alcohol continuously decreased compared to the initial wine. In Trend II, the amounts of hexanol, 2,3-butanediol, and glycerol significantly increased, reaching higher concentration than in the initial wine at the end of ageing period. Some minor alcohols (4-methyl-1-pentano, E-3-hexenol, Z-3-hexenol, 1-heptanol, 3-methylthio-1-propanol) could be set in Trend III. In this Trend, the concentration of alcohols decrease after 30 days of ageing and increase after 60 days. 2,3-butanediol gives the wine creamy and buttery notes [27] which are improved by the ageing with untoasted and light toasted oak chips. Acid-catalysed ester hydrolysis may explain the increasing amounts of these alcohols during ageing [31]. The minor alcohols were significantly dependent on time, oak chips type and ageing method. In the analysed wines, was quantified one sulphur compound, namely 3-(methylthio)-1-propanol. This compound is the end product of the deamination of methionine via the Ehrlich pathway. In initial wine, the concentration of 3-(methylthio)-1-propanol was $836 \mu \mathrm{g} / \mathrm{L}$, which significantly decrease after 90 days of ageing to $515 \mu \mathrm{g} / \mathrm{L}$ (untoasted oak chips), to $363 \mu \mathrm{g} / \mathrm{L}$ (light toasted chips), and to $483 \mu \mathrm{g} / \mathrm{L}$ (untoasted barrels). During ageing, the decrease of 3-(methylthio)-1-propanol could be caused by $\alpha$-keto acid 3-(methylthio)-propanoic acid decarboxylation and then reducing to alcohol [32]. Although considered as undesirable compounds, low concentrations of higher alcohols $(<300 \mathrm{mg} / \mathrm{L})$ can contribute to the increase of wine aroma complexity [33].

\subsubsection{Carbonyls/Volatile Aldehydes}

In the carbonyl group, only acetoin was determined. Acetoin is formed during fermentation by the microbial activity of lactic acid bacteria and yeasts [34]. Acetoin amounts increased significantly in wines aged with both variants of oak chips (untoasted and light toasted, respectively) and in the barrel-aged wine was significantly dependent on all three factors (duration, oak chips type, and ageing method) (Table 2). In initial wine, the levels of acetoin were $72 \mathrm{mg} / \mathrm{L}$, whereas, after 30 days increased to $91 \mathrm{mg} / \mathrm{L}$ (untoasted oak chips), $87 \mathrm{mg} / \mathrm{L}$ (light toasted oak chips) and $84 \mathrm{mg} / \mathrm{L}$ (untoasted barrel). 
After 90 days of ageing, the concentrations of acetoin were $151 \mu \mathrm{g} / \mathrm{L}$ (untoasted chips), $130 \mathrm{mg} / \mathrm{L}$ (light toasted chips) and $197 \mathrm{mg} / \mathrm{L}$ (untoasted barrel).

According to our results, the wine samples aged in barrels contained higher amounts of acetoin, compared to wines aged in the presence of oak chips. At higher levels of aeration, large quantities of acetoin are produced due to the redox potential of the oak barrel, which is an important factor in controlling the decomposition of the acetohydroxy acids [35]. Aeration conditions that favour the accumulation of acetoin also increase the formation of higher alcohols (isobutyl alcohol and isoamyl alcohol) [34].

\subsubsection{Carboxylic Acids}

Carboxylic acids, namely fatty acids may impact the sensory characteristics by unpleasant notes (cheesy, fatty, rancid) [36], but their flavour is essential to the aromatic equilibrium of wines [37]. Isovaleric acid present in wine samples could be synthesized from $\alpha$-keto acids. This compound, recognized by its blue cheese note [38], had a significant increase during ageing. Butanoic, octanoic, decanoic, and hexanoic acids may derive from lipid metabolism [39]. As shown in Table 2, octanoic acid had the highest level of this group, with concentrations ranging between $3267 \mu \mathrm{g} / \mathrm{L}$ in the initial wine to its highest level in untoasted barrel-aged for 60 days $(3279 \mu \mathrm{g} / \mathrm{L})$. The concentrations of all fatty acids were significantly dependent on the type of chips, duration, and method of ageing. Between fatty acids, during ageing, most of them had an increasing trend (butanoic, hexanoic, isovaleric, lactic, octanoic, E-2-hexenoic, and 2-oxoapidic acids) while others, had a decreasing trend (decanoic, hexadecanoic and pydolic acids). The loss of volatile acids and esterification reactions between alcohols and acids during the ageing, could decrease the amounts of acids [40]. In initial wine, pydolic acid was in concentration of $93 \mu \mathrm{g} / \mathrm{L}$. The loss of pydolic acid during ageing follows the same trend as their oxidative character, suggesting that this compound could contribute to wines sensory instability during chips and barrel ageing [41]. Lactic acid had a significant increase during ageing. This compound is recognized as being a softer smell contributor to wine [42]. After 90 days of barrel ageing, the concentration of lactic acid in wines treated with untoasted oak chips was $367 \mu \mathrm{g} / \mathrm{L}, 349 \mu \mathrm{g} / \mathrm{L}$ with light toasted chips, and $548 \mu \mathrm{g} / \mathrm{L}$ in untoasted barrel aged wine. The microflora that could be present in untoasted barrel, may be responsible for the higher amounts of lactic acid in barrel-aged wines [42]. The use of short ageing (30 days) time with oak chips and in barrel, respectively, gave more complexity to wines, but with a longer ageing period (90 days), the fatty acids begin to cover the other volatile aroma compounds of Muscat.

\subsubsection{Esters}

Wine esters are highly positive contributors to wine aroma. Despite alcoholic fermentation, many esters are formed by slow chemical esterification between alcohol and acids during different methods of wine ageing [43]. In wines, they contribute to flavour attributes, such as fruity, floral, and sweet notes [44]. A total of 16 esters were identified in wine samples (Table 2). Ethyl lactate, significantly increases after ageing, against the concentration from initial wine $(588 \mu \mathrm{g} / \mathrm{L})$. After ageing for 90 days, the concentration of ethyl lactate increased in wine aged with untoasted oak chips, light toasted oak chips and untoasted barrel to $1918 \mu \mathrm{g} / \mathrm{L}, 1352 \mu \mathrm{g} / \mathrm{L}$, and $1897 \mu \mathrm{g} / \mathrm{L}$, respectively. The concentration of ethyl lactate fluctuated among ageing methods, with significant difference observed between wines treated with the untoasted and toasted medium. The increased values of ethyl lactate in wines sample from untoasted chips and barrel could be produced by lactic acid bacteria which may be present in higher amounts in this medium. Ethyl lactate contributes to increasing wine aroma characteristics during ageing, enhancing aged wines a buttery and overripe fruity note [45]. Concentrations of ethyl acetate, ethyl lactate, and diethyl succinate were found to be dependent on ageing duration, oak chips type and ageing method applied. The acetates of higher alcohols detected in wine samples were isoamyl acetate, hexyl acetate, phenyl acetate, trimethylene acetate, and citronellol acetate. Among them, isoamyl acetate, produced through the esterification reactions between isoamyl alcohol and acetic acid, presented the highest amounts in initial wine of $1223 \mu \mathrm{g} / \mathrm{L}$. The isoamyl 
acetate gives a powerful banana scent [38]. After 30 days of ageing, it significantly increases to $1973 \mu \mathrm{g} / \mathrm{L}$ (untoasted oak chips), $1995 \mu \mathrm{g} / \mathrm{L}$ (light toasted chips), and to $1980 \mu \mathrm{g} / \mathrm{L}$ (in untoasted barrels). After 90 days of ageing, the concentrations in all wine samples increased over $2000 \mu \mathrm{g} / \mathrm{L}$, interestingly, with higher amounts in oak chips treated wines (Table 2). The appearance of this compound depended on all factors (ageing duration, chips types and ageing method). Phenyl acetate which gives wines a rose, fruity and tobacco note [30], was detected in higher amounts after ageing, compared with the initial wine $(228 \mu \mathrm{g} / \mathrm{L})$. According to Table 2, this compound recorded higher amounts in untoasted oak chips $(730 \mu \mathrm{g} / \mathrm{L})$ and untoasted barrel $(623 \mu \mathrm{g} / \mathrm{L})$ wine samples. Among the acetates, only hexyl acetate, previously reported as presenting apple, cherry, pear, and floral notes [15], is decreasing during ageing, regardless of the ageing method. The group of ethyl esters of carboxylic acids comprise ethyl hexanoate, ethyl octanoate, ethyl decanoate, and ethyl 3-hydroxybutanoate. Those esters showed a significantly increasing trend during ageing, regardless of the method, in the end, reaching concentrations higher compared to initial wine. According to previous studies ethyl hexanoate (pineapple, pear, floral notes) and ethyl octanoate (sweet, fruity and brandy) have low odour threshold, $5 \mu \mathrm{g} / \mathrm{L}$ and, $2 \mu \mathrm{g} / \mathrm{L}$, respectively, so their fluctuation in wine could be easily perceived [30]. Among these two esters, only ethyl hexanoate showed a decreasing trend during ageing, reaching concentrations below the initial wine. Other esters, such as diethyl malate and diethyl succinate, increased after ageing, reaching amounts significantly higher than initial wine $(33 \mu \mathrm{g} / \mathrm{L}$ and $116 \mu \mathrm{g} / \mathrm{L}$, respectively). Like ethyl lactate, higher concentrations were detected in wines aged with untoasted chips and in the untoasted barrel. The molecule size of water being smaller compared to other compounds, the water passed through wood pores (barrel or chips) more easily. The increased concentration of some compounds during ageing, might be due evaporation of the water [46]. Increasing of some esters during ageing (barrel or chips) was also previously reported $[28,47,48]$.

\subsubsection{Lactones}

Lactones are a sub-group of esters formed by internal esterification between the carbonyl and hydroxyl groups, resulting in cyclic compounds [49]. The content of butyrolactone depends on the grape variety and maceration [50]. This compound is derived from amino or organic acids during fermentation. Moreover, this compound also comes from oak, as an additional source of lactone and its presence in wines may be an indicator of wine oak ageing [51]. In this study, were identified three lactones: butyrolactone, pantolactone, and 3-4-dimethyl-2(5)-furanone. Butyrolactone had higher concentrations after ageing, with significantly increase compared with the initial wine sample $(83 \mu \mathrm{g} / \mathrm{L})$. After 90 days of ageing, the concentration of this compound significantly increased in wines samples aged with untoasted oak chip to $320 \mu \mathrm{g} / \mathrm{L}$, and untoasted barrels to $349 \mu \mathrm{g} / \mathrm{L}$, compared with wine samples aged with light toasted oak chips $(214 \mu \mathrm{g} / \mathrm{L})$. This could be explained by the fact that lactones, formed from wood lipids, can be degraded during the intense toasting of the wood. They present higher concentration in the untoasted wood or at the beginning of the toasting process [52]. Butyrolactone imprints fruity, caramel, coconut, woody, creamy, and peachy notes [53]. It significantly depended on the ageing duration and method, as well on chips type. Pantolactone was dependent on ageing duration and chips types, its concentration varying between 19 to $29 \mu \mathrm{g} / \mathrm{L}$. The higher amount of lactones could be quantified in wines after longer periods of ageing ( 6 to 12 months) [21].

\subsubsection{Terpenes}

The characteristic varietal aroma of Muscat and other aromatic wines such as Gewürztraminer is given by terpenes [31]. Monoterpenes are formed in the plant, by the fusion of two molecules of isopentenyl pyrophosphate and subsequent enzymatic reaction. The most interesting terpenes are linalool, HO-trienol, $\alpha$-terpineol, nerol, geraniol, rose, and nerol oxide, due to low perception thresholds, and their contribution to the floral notes of wines [54]. In the terpenes group, linalool, terpineol and trans-geraniol were identified in initial and aged wines, and their concentration was significantly dependent on ageing duration and method applied, but also on chips type. As can be 
noticed in Table 2, the general loss of terpenes is important as these compounds are responsible for the fresh, floral and fruity notes of wines [55]. A slight increase of linalool level above its concentration in the initial wine was found after 30 days of ageing $(1053 \mu \mathrm{g} / \mathrm{L}$ with untoasted oak chips and $1088 \mu \mathrm{g} / \mathrm{L}$ in untoasted barrel). The using of light toasted chips, caused the significant loss of all three terpenes quantified in wine-linalool, terpineol and trans-geraniol, compared to the other two ageing methods. The decrease of terpenes during wood ageing, may be explained by the sorption phenomena by chips and stave barrels, hypothesized by [42].

\subsubsection{Volatile Phenols}

Vanillin can be found in grapes and transferred to must and wine and is synthesized from ferulic acid by a hydratase/lyase enzyme [56]. Acetovanillone comes mainly from the thermal degradation of lignin in oak barrels [12]. The compounds of the vanillin group are significantly dependent on the method of preparation of the wood even if it comes from the same tonnellerie. During toasting, the concentrations of lignin-derived compounds (vanillin, guaiacol and eugenol) increase with temperature [57]. The vanillin content in initial wine was $20 \mu \mathrm{g} / \mathrm{L}$, but after ageing it significantly increased to $120 \mu \mathrm{g} / \mathrm{L}$, due to its extraction from wood. The level of vanillin in wine was very similar in all samples, after ageing, regardless of the time and method (Table 2). Vanillin imparts vanilla and sweet notes, but due to odour threshold of $200 \mu \mathrm{g} / \mathrm{L}$ [58], it could have a poor contribution to the aromatic profile of wine. The concentration of acetovanillone increased significantly after ageing. As it can be noticed in Table 2, in wines treated with chips (181-137 $\mu \mathrm{g} / \mathrm{L}-$ untoasted chips; $190-130 \mu \mathrm{g} / \mathrm{L}$ -light toast chips), the content of this compound was significantly higher than barrel-aged samples $(132-122 \mu \mathrm{g} / \mathrm{L})$. This is in accordance with other results, which found that acetovanillone best enable wine to be differentiated depending on barrel/oak chips ageing method applied [51]. The acetovanillone impart to wine vanilla note [59], but during ageing it decreases, regardless of the method. Other studies proved the discontinuous trend of acetovanillone during longer ageing periods $(4-10$ months $)[51,60]$. Glycosylated precursors from grapes formed during fermentation represent a source of p-vinyl guaiacol in wine [61]. Moreover, p-vinyl guaiacol is formed by the degradation of lignin during the toasting process. It confers smoky notes and is an indicator of the relative toast level of wood material [57]. In samples treated with light toasted oak chips, p-vinylguaiacol concentrations were $126 \mu \mathrm{g} / \mathrm{L}$ (after 30 days), with a significant increase to $156 \mu \mathrm{g} / \mathrm{L}$ (after 90 days). In wine samples aged in untoasted medium, the amount of p-vinyl guaiacol was significantly lower (Table 2). The concentrations of p-vinyl guaiacol were significantly dependent on the ageing duration and method. Guaiacol is described as a smoked, ashy, spice and roasted peanut and at higher concentration is considered off-flavour in wine. The odour threshold of p-vinylguaiacol is relatively low $(40 \mu \mathrm{g} / \mathrm{L})$ [62], so in the aged sample, the light toasted chips could impart smoked and toasted flavour to Muscat Ottonel and cover the specific aroma of this wine. The 2,3-dihydrobenzofuran is a bioactive phytochemical, known to possess antiangiogenic properties [63]. It was identified in initial wine at a concentration of $411 \mu \mathrm{g} / \mathrm{L}$. After 90 days of ageing, the level of this compound significantly decreased, regardless the method, to $156 \mu \mathrm{g} / \mathrm{L}$ (untoasted chips), $115 \mu \mathrm{g} / \mathrm{L}$ (light toasted chips), and $135 \mu \mathrm{g} / \mathrm{L}$ (untoasted barrel), respectively. The decrease might be explained by its converting to other compounds [63].

\subsection{Multivariate Analysis}

PLSR is used in the research of wine to study the relationship between chemical analysis and sensory evaluation, flavour substances and many other variables. Figure 1 shows the chemical analysis results of the Muscat Ottonel wines overlaid over the 10 different methods and periods of ageing, with the wines projected on to that space. In Figure 1, the distance between the variable and the centre of the circle shows the interpretive degree of the principal components to the variable. The correlation model of the Muscat Ottonel wine using PLSR was established to determine the influence of methods and duration of ageing on volatile compounds. PLSR applied to volatile compounds showed that method and ageing duration could be well correlated to volatile profile of wines. 


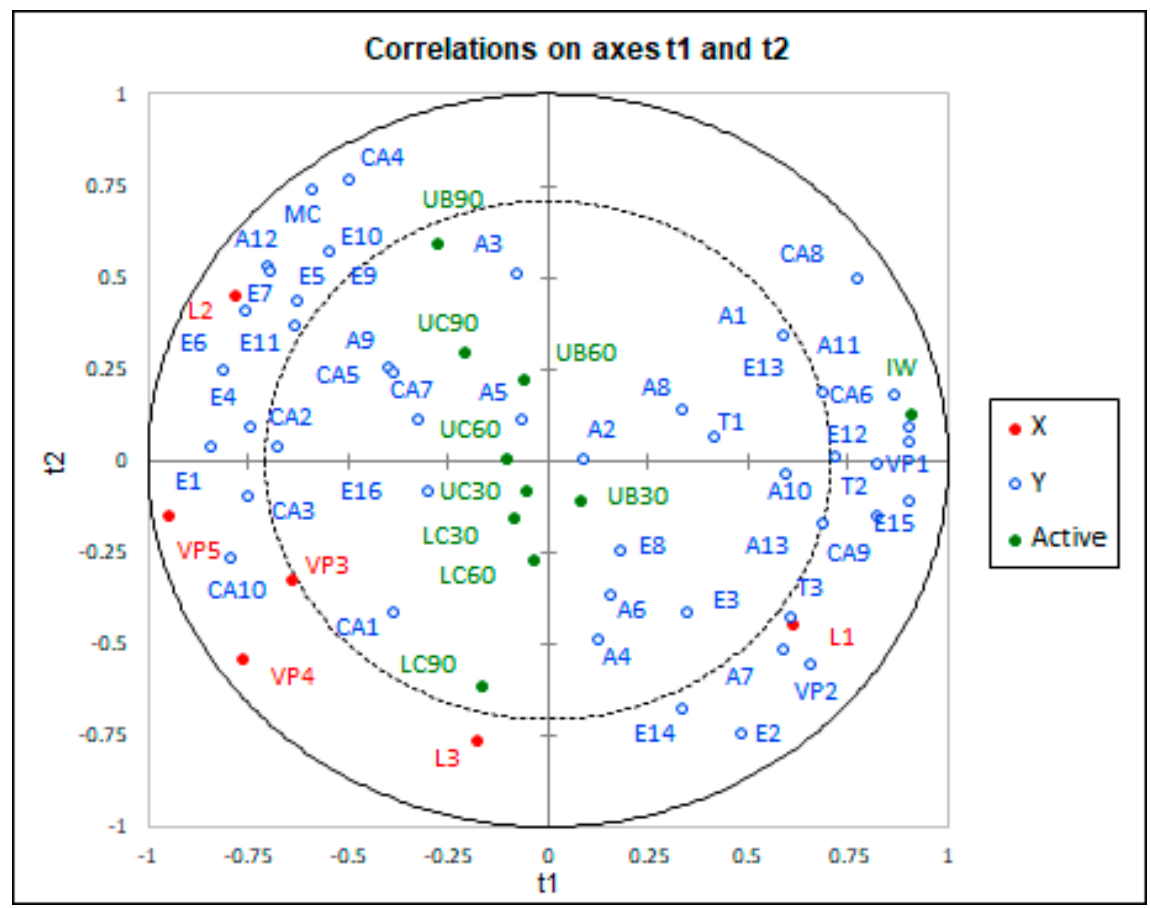

Figure 1. Partial least squares regression (PLSR) analysis, chemical data and the correlation of Muscat Ottonel wines between ageing method and period ( $\mathrm{t} 1$, chemical components of initial wine; $\mathrm{t} 2$, chemical components released from wood). IW, UC30, UC60, UC90, LC30, LC60, LC90, UB30, UB60 and UB90 are the abbreviations for 10 wines of Muscat Ottonel (IW-initial wine, UC — untoasted chips, LC — light toasted chips, UB — untoasted barrel; 30, 60 and 90—number of days of ageing). A1 (isobutanol); A2 (isoamyl alcohol); A3 (1-hexanol); A4 (4-methyl-1-pentanol); A5 (E-3-hexenol); A6 (z-3-hexenol); A7 (2-nonanol); A8 (1 heptanol); A9 (2,3-butanediol); A10 (3-methylthio-1-propanol); T1(linalool); T2 (terpineol); A11(2-phenylethanol); T3 (trans-geraniol); A12 (glycerol); A13 (benzyl alcohol); MC (acetion); E1 (isoamyl acetate); E2 (hexyl-acetate); E3 (ethyl hexanoate); E4 (ethyl lactate); E5 (ethyl octanoate); E6 (ethyl 3-hydroxybutanoate); E7 (ethyl decanoate); E8 (monoethyl-succinate); E9 (phenethyl-acetate); E10 (diethyl malate); E11 (diethyl succinate); E12 (trimethylene acetate); E13 (ethyl glycinate); E14(citronellol acetate); E15 (ethyl-4-hydroxy-butanoate); CA1 (butanoic acid); CA2 (hexanoic acid); CA3 (isovaleric acid); CA4 (lactic acid); CA5 (octanoic acid); CA6 (decanoic acid); CA7 (E-2-hexenoic acid); CA8 (hexadecanoic acid); CA9 (pydolic acid); CA10 (2-oxoapidic acid); E16 (diethyl 2-hydroxy-3-methylsuccinate); VP1 (2,3-dihydrobenzofurane); VP2 (methyl-hydroxycinnamate); L1 (3,4-dimethyl-2(5H)-furanone); L2 (butyrolactone); L3 (pantolactone); VP3 (p-vynil guaiacol); VP4 (acetovanillone); VP5 (vanillin).

As shown in Figure 1, the aroma compounds of the initial wine can be used to clearly distinguish the aroma compounds of the aged wines. Initial wine is well related to alcohols, such as isobutanol (A1), phenylethanol (A11), 1-heptanol (A8), 3 methylthio-propanol (A10), benzyl alcohol (A3), and to terpenes, such as linalool (T1) and terpineol (T2). Those compounds are responsible for the fresh and specific notes of Muscat Ottonel wines. The aged wines are well related to the esters, carboxylic acids, major carbonyls and volatile phenols [47]. The wines aged in untoasted medium (chips and barrel) appear as grouped against the wines aged with light toasted chips. The untoasted medium is correlated with a series of esters such as ethyl octanoate (E5), diethyl malate (E10), ethyl succinate (E11), phenethyl acetate (E9), ethyl octanoate (E7) and carboxylic acid, as lactic acid (C4), octanoic acid (CA5) and E-2-exanoic acid (CA7). The esters could display and enhance the fruity aroma in aged wines [28]. The butyrolactone (L2), which mainly comes from untoasted wood, is highly related to the untoasted medium and the ageing duration. Separately, the compounds that came from toasted wood (the majority of volatile phenols) are correlated with wines aged with light toasted chips. The main volatile phenols which come from thermal degradation of wood are vanillin (VP5), p-vinyl guaiacol 
(VP3) and acetovanillone (VP4) [57]. The toasted wood components from wines aged with light toasted chips, which give the vanilla and roasted aroma, are opposite to the aromatic compounds from unaged wines with floral and fresh notes. On the respect of the ageing duration, in the Figure 1 it may be seen some similarities between wines aged for 30 days, regardless of the method of ageing. After 60 days of ageing, there are some clear differences between wines aged in barrel or with chips, especially with light toasted chips, from the volatile compounds point of view. After 90 days of ageing, the aromatic compounds of wine treated with untoasted chips and barrels were almost similar. According to this, it can be said that it can obtain almost the same wine aged with untoasted chips and untoasted barrel, but in a cost-efficient manner.

\section{Conclusions}

Muscat Ottonel is a part of a family of grape varieties which produce floral and fruity fragrant wines. The chemical composition and volatile profile varied significantly according to the period and method of ageing, but also based on chips types being used. The physicochemical variables of the wine, especially volatile and titratable acidity and alcohol content, are important parameters influenced by time and ageing factors. Several wine compounds concentration, such as alcohols, esters and terpenes, decrease after short ageing and the loss of wine aroma may occur. Interestingly, the untoasted medium enhanced the concentration of linalool after 30 days of ageing. The higher concentrations of ageing compounds (acetovanillone and p-vinyl guaiacol) were quantified in wine samples treated with light toasted chips. Multivariate analysis clearly separated the unaged wines from those aged in different methods, and also the wines aged with toasted and untoasted medium. Oak aged wines may be distinguished due to the content of acetovanillone, vanillin, p-vinylguaiacol, and butyrolactone. Ageing with oak chips and in barrels cause degradation and loss of some volatile compounds, but also leads to the formation of new oak-related ones. The oak chips ageing method could enhance the volatile profile complexity of Muscat Ottonel wines at the same level as barrels, but as a less expensive alternative. Based on these results, the winemaker could predict, to a certain level, the impact of oak chips and barrel ageing on the wine volatile profile, by choosing the appropriate toast level and the period of ageing. Improving wines quality is congruent with consumer demands, but also with the wine price.

Author Contributions: Conceptualization, A.C., T.E.C. and E.G.; methodology, C.R.P., T.I.P., and A.C.B.; software, C.R.P.; validation, A.C., C.I.B., M.M., and E.G.; formal analysis, M.M. and A.C.; investigation, T.E.C.; resources, A.C., C.I.B., and E.G.; data curation, E.G.; writing - original draft preparation, A.C. and E.G.; writing-review and editing, T.E.C.; visualization, A.C.; supervision, T.E.C. and E.G.; project administration, A.C., T.I.P., and A.C.B. All authors have read and agreed to the published version of the manuscript.

Funding: The publication was supported by funds from the National Research Development Projects to finance excellence (PFE)-37/2018-2020 granted by the Romanian Ministry of Research and Innovation.

Conflicts of Interest: The authors declare no conflict of interest.

\section{References}

1. Salanţă, L.C.; Tofană, M.; Pop, C.; Pop, A.; Coldea, T.; Mudura, E. Beverage alcohol choice among university students: Perception, consumption and preferences. Bull. Univ. Agric. Sci. Vet. Med. Cluj-Napoca Food Sci. Technol. 2017, 74, 23-29. [CrossRef]

2. Alañón, M.E.; Díaz-Maroto, M.C.; Pérez-Coello, M.S. New strategies to improve sensorial quality of white wines by wood contact. Beverages 2018, 4, 91. [CrossRef]

3. Higgins, L.M.; Llanos, E. A healthy indulgence? Wine consumers and the health benefits of wine. Wine Econ. Policy 2015, 4, 3-11. [CrossRef]

4. King, E.S.; Johnson, T.E.; Bastian, S.E.P. Consumer liking of white wines: Segmentation using self-reported wine liking and wine knowledge. Int. J. Wine Bus. Res. 2012, 24, 33-46. [CrossRef] 
5. Culbert, J.A.; Ristic, R.; Ovington, L.A.; Saliba, A.J.; Wilkinson, K.L. Influence of production method on the sensory profile and consumer acceptance of Australian sparkling white wine styles. Aust. J. Grape Wine R. 2017, 23, 170-178. [CrossRef]

6. Coldea, T.E.; Socaciu, C.; Mudura, E.; Socaci, S.A.; Ranga, F.; Pop, C.R.; Vriesekoop, F.; Pasqualone, A. Volatile and phenolic profiles of traditional Romanian apple brandy after rapid ageing with different wood chips. Food Chem. 2020, 320, 126643. [CrossRef]

7. Frangipane, M.T.; De Santis, D.; Ceccarelli, A. Influence of oak woods of different geographical origins on quality of wines aged in barriques and using oak chips. Food Chem. 2007, 103, 46-54. [CrossRef]

8. Ianni, F.; Segoloni, E.; Blasi, F.; Di Maria, F. Low-molecular-weight phenols recovery by eco-friendly extraction from Quercus spp. wastes: An analytical and biomass-sustainability evaluation. Processes 2020, 8, 387. [CrossRef]

9. Fernández de Simón, B.; Martínez, J.; Sanz, M.; Cadahía, E.; Esteruelas, E.; Muñoz, A.M. Polyphenolic compounds as chemical markers of wine ageing in contact with cherry, chestnut, false acacia, ash and oak wood. Food Chem. 2014, 147, 346-356. [CrossRef]

10. Chinnici, F.; Natali, N.; Bellachioma, A.; Versari, A.; Riponi, C. Changes in phenolic composition of red wines aged in cherry wood. LWT-Food Sci. Technol. 2015, 60, 977-984. [CrossRef]

11. Gortzi, O.; Metaxa, X.; Mantanis, G.; Lalas, S. Effect of artificial ageing using different wood chips on the antioxidant activity, resveratrol and catechin concentration, sensory properties and colour of two Greek red wines. Food Chem. 2013, 141, 2887-2895. [CrossRef] [PubMed]

12. Canas, S. Phenolic composition and related properties of aged wine spirits: Influence of barrel characteristics. A review. Beverages 2017, 3, 55. [CrossRef]

13. Caldeira, I.; Anjos, O.; Belchior, A.P.; Canas, S. Sensory impact of alternative ageing technology for the production of wine brandies. Ciência Téc. Vitiv. 2017, 32, 12-22. [CrossRef]

14. Vararu, F.; Moreno-García, J.; Niculaua, M.; Cotea, V.V.; Mayén, M.; Moreno, J. Fermentative volatilome modulation of Muscat Ottonel wines by using yeast starter cultures. LWT-Food Sci. Technol. 2020, 129, 109575. [CrossRef]

15. Caven-Quantrill, J.; Buglass, A.J. Analysis of volatile components of varietal English wines using stir bar sorptive extraction/gas chromatography-mass spectrometry. Beverages 2017, 3, 62. [CrossRef]

16. Aleixandre, J.L.; Padilla, A.I.; Navarro, L.L.; Suria, A.; García, M.J.; Álvarez, I. Optimization of making barrel-fermented dry Muscatel wines. J. Agric. Food Chem. 2003, 51, 1889-1893. [CrossRef]

17. Bora, F.D.; Donici, A.; Oşlobanu, A.; Fițiu, A.; Babeș, A.C.; Bunea, C.I. Qualitative assessment of the white wine varieties grown in Dealu Bujorului vineyard, Romania. Not. Bot. Horti. Agrobo. 2016, 44, 593-602. [CrossRef]

18. Cabredo-Pinillos, S.; Cedrón-Fernández, T.; Sáenz-Barrio, C. Comparison of different extraction methods applied to volatile compounds in wine samples previous to the determination by gas chromatography. Anal. Lett. 2004, 37, 3063-3084. [CrossRef]

19. Mamede, M.E.O.; Pastore, G.M. Study of methods for the extraction of volatile compounds from fermented grape must. Food Chem. 2006, 96, 586-590. [CrossRef]

20. Andujar-Ortiz, I.; Moreno-Arribas, M.V.; Martín-Álvarez, P.J.; Pozo-Bayón, M.A. Analytical performance of three commonly used extraction methods for the gas chromatography-mass spectrometry analysis of wine volatile compounds. J. Chromatogr. A 2009, 1216, 7351-7357. [CrossRef]

21. Manolache, M.; Pop, T.I.; Babeș, A.C.; Farcaș, I.A.; Muncaciu, M.L.; Gal, E.; Călugăr, A. Volatile composition of some red wines from Romania assessed by GC-MS. Studia UBB Chem. 2018, LXIII, 125-142. [CrossRef]

22. Antoce, A.O.; Călugăru, L.L. Evolution of grapevine surfaces in Romania after accession to European Union period 2007-2016. In Proceedings of the BIO Web of Conferences, 40th World Congress of Vine and Wine, Sofia, Bulgaria, 29 May-2 June 2017; p. 03018.

23. González-Barreiro, C.; Rial-Otero, R.; Cancho-Grande, B.; Simal-Gándara, J. Wine aroma compounds in grapes: A critical review. J. Crit. Rev. Food Scien. Nutr. 2015, 55, 202-218. [CrossRef]

24. Stoica, F.; Muntean, C.; Băducă, C.; Popescu Mitroi, I. Differences in Muscat wine aroma composition depending maceration and fermentation processes. Rom. Biotech. Lett. 2015, 20, 10343-10351.

25. Balcerek, M.; Pielech-Przybylska, K.; Dziekońska-Kubczak, U.; Patelski, P.; Strąk, E. Changes in the chemical composition of plum distillate during maturation with oak chips under different conditions. Food Technol. Biotechnol. 2017, 55, 333-359. [CrossRef] 
26. Rodríguez-Rodríguez, P.; Gómez-Plaza, E. Dependence of oak-related volatile compounds on the physicochemical characteristics of barrel-aged wines. Food Technol. Biotechnol. 2012, 50, 59-65.

27. Bao, J.; Zhang, Z. Volatile compounds of young wines from cabernet sauvignon, cabernet gernischet and chardonnay varieties grown in the Loess plateau region of China. Molecules 2010, 15, 9184-9196. [CrossRef]

28. Dumitriu (Gabur), G.D.; Teodosiu, C.; Gabur, I.; Cotea, V.V.; Peinado, R.A.; López de Lerma, N. Evaluation of aroma compounds in the process of wine ageing with oak chips. Foods 2019, 8, 662. [CrossRef] [PubMed]

29. Antonelli, A.; Castellari, L.; Zambonelli, C.; Carnacini, A. Yeast influence on volatile composition of wines. J. Agric. Food Chem. 1999, 47, 1139-1144. [CrossRef]

30. Furdíková, K.; Makyšová, K.; Špánik, I. Effect of indigenous S. cerevisiae strains on higher alcohols, volatile acids and esters in wine. Czech. J. Food Sci. 2017, 35, 131-142. [CrossRef]

31. Rapp, A.; Mandery, H. Wine aroma. Experientia 1986, 42, 873-884. [CrossRef]

32. Darriet, P.; Pons, M.; Henry, R.; Dumont, O.; Findeling, V.; Cartolaro, P.; Calonnec, A.; Dubourdieu, D. Impact odorants contibuting to the Fungus type aroma from grape berries contaminated by powdery mildew (Uncinula necator); incidence of enzymatic activities of the yeast Saccharomyces cerevisiae. J. Agric. Food Chem. 2002, 50, 3277-3282. [CrossRef] [PubMed]

33. Marcon, A.R.; Schwarz, L.V.; Dutra, S.V.; Delamare, A.P.L.; Gottardi, F.; Parpinello, G.P.; Echeverrigaray, S. Chemical composition and sensory evaluation of wines produced with different Moscato varieties. In Proceedings of the BIO Web of Conferences, 41st World Congress of Vine and Wine, Punta del Este, Uruguay, 19-23 November 2018; Volume 12, p. 02033. Available online: https://doi.org/10.1051/bioconf/20191202033 (accessed on 9 April 2020).

34. Romano, P.; Suzzi, G. Origin and production of acetoin during wine yeast fermentation. Appl. Environ. Microbiol. 1996, 62, 309-315. [CrossRef] [PubMed]

35. White, F.H.; Wainwright, T. Analysis of diacetyl and related compounds in fermentations. J. Inst. Brew. 1975, 81, 37-45. [CrossRef]

36. Rocha, M.S.; Rodrigues, F.; Coutinho, P.; Delgadillo, I.; Coimbra, A.M. Volatile composition of Baga red wine: Assessment of the identification of the would-be impact odourants. Anal. Chim. Acta 2004, 51, 257-262. [CrossRef]

37. Etiévant, P.X. Wine. In Volatile Compounds in Foods and Beverages, 1st ed.; Maarse, H., Ed.; Marcel Dekker, Inc.: New York, NY, USA, 1991; Volume 14, pp. 483-546.

38. Louw, L.; Tredoux, A.G.J.; Van Rensburg, P.; Kidd, M.; Naes, T.; Nieuwoudt, H.H. Fermentation-derived aroma compounds in varietal young wines from South Africa. S. Afr. J. EnoL Vitic. 2010, 31, 213-225. [CrossRef]

39. Dubois, P. Les arômes des vins et leurs defauts (cont). Revue Française d'Enologie. Dugelay 1994, 145, 27-40.

40. Xu, M.L.; Yu, Y.; Ramaswamy, H.S.; Zhu, S.M. Characterization of Chinese liquor aroma components during aging process and liquor age discrimination using gas chromatography combined with multivariable statistics. Sci. Rep. 2017, 7, 39671. [CrossRef] [PubMed]

41. Nikolantonaki, M.; Julien, P.; Coelho, C.; Roullier-Gall, C.; Ballester, J.; Schmitt-Kopplin, P.; Gougeon, R.D. Impact of glutathione on wines oxidative stability: A combined sensory and metabolomic study. Front. Chem. 2018, 6, 182. [CrossRef] [PubMed]

42. Garde-Cerdan, T.; Ancín-Azpilicueta, C. Review of quality factors on wine ageing in oak barrels. Trends Food Sci. Technol. 2006, 17, 438-447. [CrossRef]

43. Sumby, K.M.; Grbin, P.R.; Jiranek, V. Microbial modulation of aromatic esters in wine: Current knowledge and future prospects. Food Chem. 2010, 121, 1-16. [CrossRef]

44. Diaz-Maroto, M.C.; Schneider, R.; Baumes, R. Formation pathways of ethyl esters of branched short-chain fatty acids during wine aging. J. Agric. Food Chem. 2005, 53, 3503-3509. [CrossRef]

45. Pérez-Coello, M.S.; Sánchez, M.A.; García, E.; González-Viñas, M.A.; Sanz, J.; Cabezudo, M.D. Fermentation of white wines in the presence of wood chips of American and French oak. J. Agric. Food Chem. 2000, 48, 885-889. [CrossRef]

46. Herrera, P.; Durán-Guerrero, E.; Sánchez-Guillén, M.M.; García-Moreno, M.V.; Guillén, D.A.; Barroso, C.G.; Castro, R. Effect of the type of wood used for ageing on the volatile composition of Pedro Ximénez sweet wine. J. Sci. Food. Agric. 2020, 100, 2512-2521. [CrossRef] [PubMed] 
47. Dumitriu, G.D.; Lopez de Lerma, N.; Zamfir, C.I.; Cotea, V.V.; Peinado, R.A. Volatile and phenolic composition of red wines subjected to aging in oak cask of different toast degree during two periods of time. LWT-Food Sci. Technol. 2017, 86, 643-651. [CrossRef]

48. Jiménez-Moreno, N.; Ancín-Azpilicueta, C. The development of esters in filtered and unfiltered wines that have been aged in oak barrels. Int. J. Food Sci. Technol. 2006, 41, 155-161. [CrossRef]

49. Pérez-Olivero, S.J.; Pérez-Pont, M.L.; Conde, J.E.; Pérez-Trujillo, J.P. Determination of lactones in wines by headspace solid-phase microextraction and gas chromatography coupled with mass spectrometry. J. Anal. Methods Chem. 2014, 2014, 1-10. [CrossRef]

50. Bueno, J.E.; Peinado, R.; Moreno, J.; Medina, M.; Moyano, L.; Zea, L. Selection of volatile aroma compounds by statistical and enological criteria for analytical differentiation of musts and wines of two grape varieties. J. Food Sci. 2003, 68, 158-163. [CrossRef]

51. Hernández-Orte, P.; Franco, E.; González Huerta, C.; Martínez García, J.; Cabellos, M.; Suberviola, J.; Orriols, I.; Cacho, J. Criteria to discriminate between wines aged in oak barrels and macerated with oak fragments. Food Res. Int. 2014, 57, 234-241. [CrossRef]

52. Giménez-Martínez, R.; López-García de la Serrana, H.; Villalón-Mir, M.; Quesada-Granados, J.; López-Martínez, M.C. Influence of wood heat treatment, temperature and maceration time on vanillin, syringaldehyde, and gallic acid contents in oak word and wine spirit mixtures. Am. J. Enol. Viticult. 1996, 47, 441-446.

53. Perestrelo, R.; Fernandes, A.; Albuquerque, F.; Marques, J.C.; Câmara, J. Analytical characterization of the aroma of Tinta Negra Mole red wine: Identification of the main odorants compounds. Anal. Chim. Acta 2006, 563, 154-164. [CrossRef]

54. Ribéreau-Gayon, P.; Glories, Y.; Maujean, A.; Dubourdieu, D. Varietal aroma. In Handbook of Enology Volume 2-The Chemistry of Wine and Stabilization and Treatments, 2nd ed.; Ribéreau-Gayon, P., Glories, Y., Maujean, A., Dubourdieu, D., Eds.; John Wiley and Sons Ltd.: Chichester, UK, 2000; pp. 187-206.

55. Del Caro, A.; Piombino, P.; Genovese, A.; Moio, L.; Fanara, C.; Piga, A. Effect of bottle storage on colour, phenolics and volatile composition of Malvasia and Moscato white wines. S. Afr. J. Enol. Vitic. 2014, 35, 128-138. [CrossRef]

56. Gallage, N.J.; Hansen, E.H.; Kannangara, R.; Olsen, C.E.; Motawia, M.S.; Jørgensen, K.; Holme, I.; Hebelstrup, K.; Grisoni, M.; Møller, B.L. Vanillin formation from ferulic acid in Vanilla planifolia is catalysed by a single enzyme. Nat. Commun. 2014, 5, 4037. [CrossRef] [PubMed]

57. Farrell, R.R.; Wellinger, M.; Gloess, A.N.; Nichols, D.S.; Breadmore, M.C.; Shellie, R.A.; Yeretzian, C. Real-time mass spectrometry monitoring of oak wood toasting: Elucidating aroma development relevant to oak-aged wine quality. Sci. Rep. 2015, 5, 17334. [CrossRef] [PubMed]

58. Pengtao, Z.; Gao, J.; Qian, M.; Li, H. Characterization of the key aroma compounds in Chinese Syrah wine by gas chromatography-olfactometry-mass spectrometry and aroma reconstitution studies. Molecules 2017, 22, 1045. [CrossRef]

59. Rodríguez-Bencomo, J.J.; Schneider, R.; Lepoutre, J.P.; Rigou, P. Improved method to quantitatively determine powerful odorant volatile thiols in wine by headspace solid-phase microextraction after derivatization. J. Chromatogr. A 2009, 1216, 5640-5646. [CrossRef]

60. Garde-Cerdán, T.; Rodríguez-Mozaz, S.; Ancín-Azpilicueta, C. Volatile composition of aged wine in used barrels of French oak and of American oak. Food Res. Int. 2002, 35, 603-610. [CrossRef]

61. Ilc, T.; Werck-Reichhart, D.; Navrot, N. Meta-analysis of the core aroma components of grape and wine aroma. Front. Plant. Sci. 2016, 7, 1472. [CrossRef]

62. Guth, H. Quantitation and sensory studies of character impact odorants of different white varieties. J. Agric. Food Chem. 1997, 45, 3027-3032. [CrossRef]

63. Dharmalingam, R.; Nazni, P. Phytochemical evaluation of Coriandrum L. flowers. Int. J. Food Nutr. Sci. 2013, 2, 34-39.

(C) 2020 by the authors. Licensee MDPI, Basel, Switzerland. This article is an open access article distributed under the terms and conditions of the Creative Commons Attribution (CC BY) license (http://creativecommons.org/licenses/by/4.0/). 